\title{
COMBINED EFFECT OF STIRRUP VOLUMETRIC RATIO WITH VERTICAL STEEL BARS ON THE BEHAVIOR OF R.C. COLUMNS UNDER AXIAL LOADS
}

\author{
Mohamed Kamel Abd-Elhamed \\ Department of Structural Engineering, Faculty of Engineering, Al-Azhar University, Cairo, \\ Egypt. \\ E-mail: mohamedabdelhamed.14@azhar.edu.eg
}

\begin{abstract}
:
Most international codes in the design of R.C. columns use only the vertical steel bar effect in determining ultimate load capacity. No combination effect of stirrup volumetric ratio with vertical steel bar has been taken in design of the R.C. column. The present study deals with an experimental and analytical analysis of the behavior of reinforced concrete columns with different percentage of vertical steel bars $(\mu)$ and stirrups volumetric ratio $(\rho \mathrm{v})$. A total of sixteen R.C. columns with cross-section $(250 \times 250 \times 1000) \mathrm{mm}$ were tested and divided into four groups and tested until failure under axial load. Group 1 consisted of four columns with vertical steel bars with $(\mu)=0.18 \%$ and stirrups $(\rho \mathrm{v})=(0.27 \%, 0.48 \%, 0.75$ and $1.09 \%)$. Group 2, 3 and 4 having same stirrups $(\rho$ v) \% with vertical bars $(\mu)=(0.32 \%, 0.50 \%, 0.75 \%)$ respectively. The test results showed that increasing in the percentage of vertical steel bars $(\mu)$ $=(0.18 \%$ up to $0.73 \%)$ give an increase of the ultimate failure load up to $9.7 \%$. Also, increasing percentage of stirrups volumetric ratio $(\rho \mathrm{v})=(0.27 \%$ up to $1.09 \%)$ gives an increase of the ultimate failure load up to $12.3 \%$ and enhancement in ductility of columns Thus, the increase in the ultimate load of columns due to stirrups effect should be taken into consideration in the design of R.C columns. The analytical analysis (FEA) models can be simulate the experimental behavior of tested columns and can be suitable to reduce time and cost.
\end{abstract}

KEYWORDS: Einforced Concrete Column, Ultimate Load Capacity, Finite Element, Axially Loaded, Vertical Bar, Stirrups.

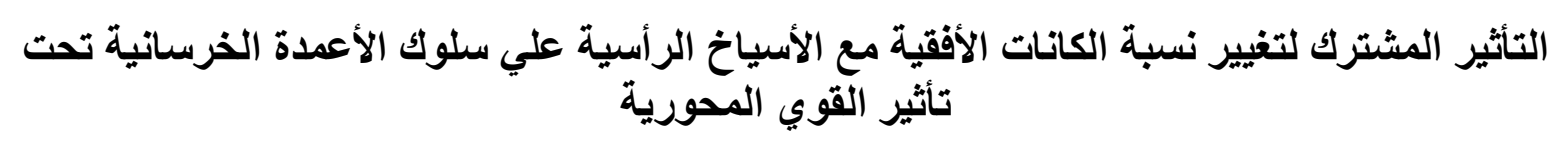

$$
\begin{aligned}
& \text { محمد كامل عبد الحميد } \\
& \text { قسم الهندسة الانشيائيه، كليه الهندسة، جامعه الأزهر ، القاهرة، مصر. } \\
& \text { البريد الالكتروني:E-mail: mohamedabdelhamed.14@azhar.edu.eg }
\end{aligned}
$$

\footnotetext{
تلخل معظم الأكو اد العالمية عند تصميم الأعمدة الخرسانية المسلحة تأثنير الحديد الرأسي فقط عند حساب الحمل الأقصىي

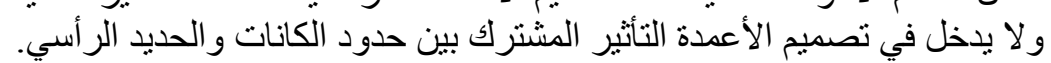

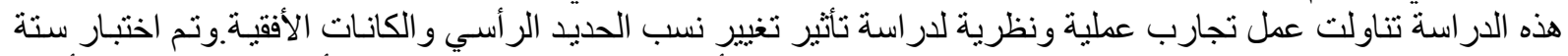

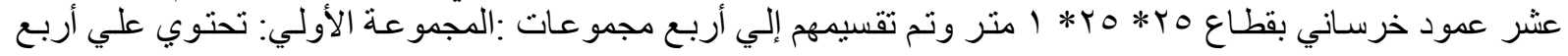




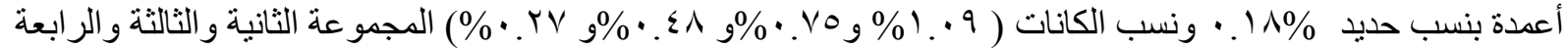

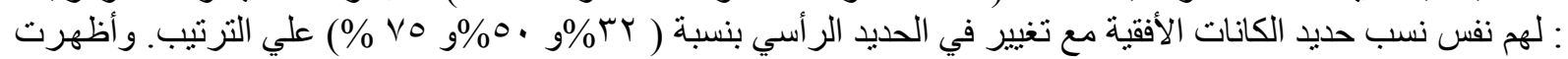

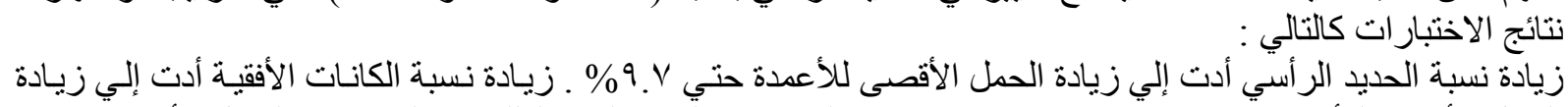

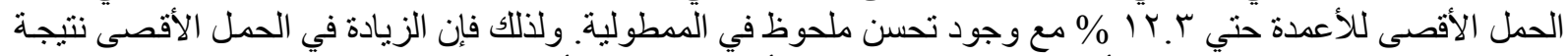

$$
\begin{aligned}
& \text { التغيير في نسب حديد الكانات يجب أن يؤخ بذ بعين الاعتبار في أعمال تصميم الأعمدة. } \\
& \text { الكلمات المفتاحية: الأعمدة الخرسانيه المسلحة، سعة التحميل القصوي، العناصر المحدودة، القوي المحورية، }
\end{aligned}
$$

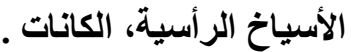

\subsection{INTRODUCTION}

One of the most significant construction components in the building which play an important role are the columns. The columns are the main structural element needed to resist several loads. In spite of many researchers investigated the different parameters that affect columns ultimate capacity were dependent on a percentage of vertical steel bars, stirrup volumetric ratio, stirrup spacing and cross-section of columns. However, most international codes neglect the contribution of stirrups effect on the ultimate capacity of RC columns. The present study deals with an experimental and analytical analysis to determine the combined effect different vertical steel bars and stirrups volumetric ratio percentage on the behavior of RC columns. Cusson, D., \& Paultre, P. (1994) [1] presented an experimental study to investigate number of variables that affect RC columns behavior, such as the transverse reinforcement ratio, the tie spacing and the longitudinal reinforcement ratio. Twenty-seven large-scale columns $(235 \times 235 \times 1400 \mathrm{~mm})$ were tested under concentric loading. The test results showed that the transverse reinforcement ratio has the most important beneficial effect on the stress-strain behavior of concrete. In addition, the lateral confining pressure imposed on the concrete core is directly related to the amount of lateral reinforcement. When the ratio of the lateral reinforcement is increased about 50\%, increases in strength is gained between 10 and $16 \%$ was obtained and enhancements in toughness is gained between 55 and $136 \%$. However, when the lateral reinforcement ratio is increased from 2.0 to $2.8 \%$, increases in strength is gained by $10 \%$ and a low enhancement in toughness $17 \%$ is obtained.

Li Bing, R. Park, and H. Tanaka (2001) [2] studied the effect of volumetric ratio and spacing of stirrups reinforcement on the behavior of RC columns. The spacing used started from 1.66 up to 5.83 diameter of longitudinal bar. The results showed that the behavior of R.C. become less ductile with increasing stirrups spacing.

Yehia A. Daou and EyadSeifeddinc (2001) [3] tested twenty-four square tied columns with cross section $12 \times 12 \mathrm{~cm}$ and height $56 \mathrm{~cm}$ under concentric load to explore the influence of the diameter and spacing of stirrups on the strength and behavior of reinforced concrete columns. The diameter of stirrups used were 2,4 and $6 \mathrm{~mm}$ and stirrups spacing ranged between $6.25 \mathrm{~cm}$ and $25 \mathrm{~cm}$. The experimental test results showed that full capacities of columns were achieved when the ratio of the stirrups area / (spacing $\mathrm{x}$ column dimension) Asv/(Sv.b) $\cong 0.1 \%$ and the volume of the stirrups / volume of concrete Vst / Vc@ $3 \%$.

J. Němečekand at el (2004) [4] tested six reinforced concrete columns with a square cross section $50 \times 150 \mathrm{~mm}$ and length $150 \mathrm{~mm}$. Three different densities of stirrups were chosen. The test results showed that the ductility and ultimate load of the columns increases as the distance between stirrups becomes smaller.

Hong, K. N., et al. (2006) [5] presented an experimental study to investigate the behavior of high-strength concrete (HSC) columns with a tie volumetric ratio smaller than $2.0 \%$.All columns were tested under concentric loading. The test variables included measurements of concrete compressive strength; measurements of tie yield strength; and the tie volumetric ratio. The results showed that the tie volumetric ratio is more effective and performed is better in case normal strength concrete.

Khaleek, A., et al. (2012) [6] studied the effect of different spacing of stirrups on the compressive strength of concrete columns. Cylindrical and square test columns having height to diameter (or width) ratio as 2 were used. The longitudinal distance between the stirrups at the middle part of the columns was ranged from 25 up to $150 \mathrm{~mm}$ and the distance of stirrups at the ends of columns was denser to prevent damage in this region. The test results showed 
that increasing ratio of volume of stirrups to the volume of core, the peak strength of concrete substantially increases.

Wasan Ismail Khalil and at el (2012) [7] investigated the effect of variation lateral confined stirrups spacing on the behavior square short concrete columns. The test results showed that with the increases in volumetric ratio (decrease spacing between stirrups) increase the confinement efficiency of concrete and decrease the lateral buckling of the longitudinal bars also, the volumetric ratios of longitudinal reinforcement is slightly affected the peak strength of columns. Radnic, J., et al (2013) [8] studied the impact of adding stirrups on both the compressive strength and ductility of axially loaded confined reinforced concrete columns having rectangular cross-section. The influence of varying concrete strengths and the difference of both stirrup bar diameters and spacing were studied on the column's ultimate capacity and ductility. It was shown that stirrups spacing greatly have more effect than the stirrup bar diameter .Columns with smaller stirrup spacing will gain greater strength capacity and greater ductility when the same quantity of transversal reinforcement per column length unit is used. Smaller stirrup bar diameter at smaller spacing is more favorable than greater stirrup bar diameter at greater spacing. Xiang Zeng (2016) [9] developed a Finite Element model to evaluate the behavior of concrete confined by stirrups in square RC columns. Xiang Zeng proposed a new uniaxial compression stress-strain relation of concrete considering the confinement effect of stirrups. Three volumetric stirrup ratios $(0.8 \%, 1.6 \%$ and $2.39 \%)$ were used. The study showed that increasing the value of stirrup ratios has little effect on the strength of confined concrete, but it improves the ductility of the confined concrete.

Min Du and at el (2017) [10] tested twenty four stocky reinforced concrete columns confined by stirrups ratios from $1.26 \%$ up to $2.89 \%$. The test results observed indicate that increasing the stirrup ratio could make the enhancement of the nominal strength and makes a less brittle failure behavior of RC columns.

Liu Jin and at el (2018) [11] tested twelve square reinforced concrete columns with two different stirrups ratios (i.e., $0 \%$ and $0.66 \%$ ) under small-eccentric compressive loading. The specimens were divided into two series to explore the influences of stirrup confinement on the size effect of RC columns under the small eccentric compressive loading. Series one: without stirrups in the middle part of the RC columns and series two: having middle stirrups with the stirrup ratio of $0.66 \%$. The test results showed that the $\mathrm{RC}$ columns without middle stirrups failed by compression-shear mode. While, the other RC columns with middle stirrups failed by localized at the middle part where a wedge-shaped pattern developed. In addition, the presence of stirrups improves the nominal strengths and makes the failure of columns less brittle.

M.K. Abd-Elhamed and M.E. Owida (2019) [12] studied the effect of stirrups densification at top and bottom of columns and along of height of columns on ultimate load capacity. The results showed that the failure load increases by increasing the percentage of stirrups densification height at top and bottom of column / total column height.

The present study deals with an experimental (EXP) and analytical analysis by Finite element (FEA) to investigate the combined effect of vertical steel bars and stirrups volumetric ratio percentage on the behavior of reinforcement concrete columns.

\section{EXPERIMENTAL PROGRAM}

\subsection{Detailed model}

A total of sixteen RC columns models have a typical cross section of $250 \mathrm{~mm} \times 250 \mathrm{~mm}$ with the height of $1000 \mathrm{~mm}$ is shown in Figure 1. All specimens contained four vertical steel bars with a deferent diameter $6,8,10$ and $12 \mathrm{~mm}$ corresponding to vertical steel bars percentage $(\mu)=(0.18,0.32,0.50$ and $0.73 \%)$ respectively. The stirrups diameters are $6,8,10$ and 12 corresponding to stirrups volumetric ratio percentage $(\rho \mathrm{v})=(0.27,0.48,0.75$ and $1.09 \%)$ respectively. The concrete cover was $25 \mathrm{~mm}$. The columns were divided into four groups as shown in Table [1]. 
COMBINED EFFECT OF STIRRUP VOLUMETRIC RATIO WITH VERTICAL STEEL BARS ON THE BEHAVIOR OF R.C. COLUMNS UNDER AXIAL LOADS

\subsection{COLUMNS CHARACTERISTICS}

\subsubsection{Used material}

- Crushed stone has a maximum nominal size of $(0.07-20.0 \mathrm{~mm})$ was used as the coarse aggregate in the mix.

- Graded sand having sizes in the range of $(0.075-0.3 \mathrm{~mm})$ was used as the fine aggregate in the mix.

- Ordinary Portland cement was used.

- Clean fresh water is used for mixing and curing the specimens. Percentage of water cement ratio $50 \%$.

- The concrete mix used in all specimens was designed according to the Egyptian code of practice.

- The concrete mix was designed to obtain target strength of $20 \mathrm{~N} / \mathrm{mm} 2$ at the age of 28 days as shown in Table [2]. The test specimens were casted in steel forms shown in Fig. [2].

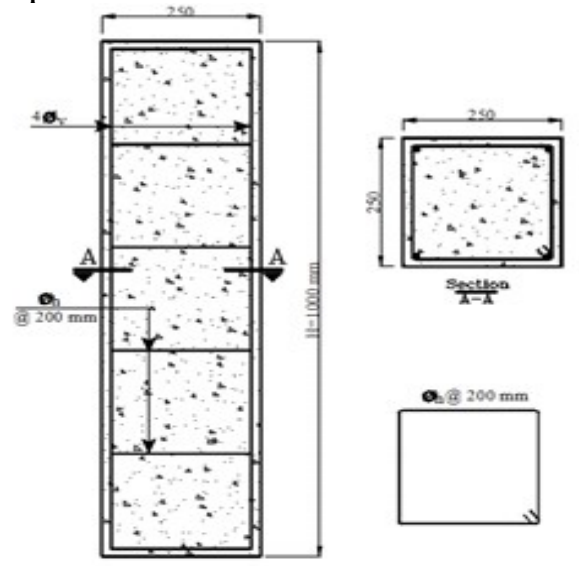

Fig. 1 Geometrical properties of column specimens

Table 1. Geometrical and properties of the column specimens

\begin{tabular}{|c|c|c|c|c|c|c|}
\hline & \multirow[t]{2}{*}{$\begin{array}{l}\text { Columns } \\
\text { name }\end{array}$} & \multirow[t]{2}{*}{$\begin{array}{c}\text { Columns } \\
\text { dimension }\end{array}$} & \multicolumn{2}{|c|}{$\begin{array}{c}\text { Internal reinforcement } \\
\text { bars diameter (mm) }\end{array}$} & \multirow{2}{*}{$\begin{array}{l}\text { Vertical } \\
\text { steel bar }(\mu) \\
\text { "\%" }\end{array}$} & \multirow[t]{2}{*}{$\begin{array}{l}\text { Stirrups volumetric } \\
\text { ratio }(\rho \text { v) \% }\end{array}$} \\
\hline & & & $\begin{array}{l}\text { Vertical } \\
\text { bar } \\
\end{array}$ & Stirrups & & \\
\hline & $\begin{array}{ll}\text { C1-1 } \\
\end{array}$ & \multicolumn{3}{|c|}{ Control Column } & $0.18 \%$ & $0.27 \%$ \\
\hline \multirow{4}{*}{$\begin{array}{l}\mathrm{Gr} \\
\text { ou } \\
\text { p } 1\end{array}$} & C1-1 & \multirow{17}{*}{ 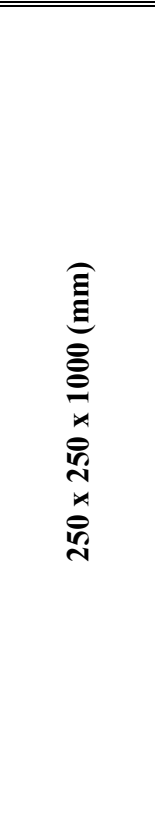 } & \multirow{4}{*}{$4 \Phi 6$} & $5 \Phi 6$ & \multirow{4}{*}{$0.18 \%$} & $0.27 \%$ \\
\hline & C1-2 & & & $5 \Phi 8$ & & $0.48 \%$ \\
\hline & C1-3 & & & $5 \Phi 10$ & & $0.75 \%$ \\
\hline & C1-4 & & & $5 \Phi 12$ & & $1.09 \%$ \\
\hline \multirow{4}{*}{$\begin{array}{l}\text { Gr } \\
\text { ou } \\
\text { D } 2\end{array}$} & $\overline{C 22-1}$ & & & $\overline{5 \Phi 6}$ & \multirow{4}{*}{$0.32 \%$} & $0.27 \%$ \\
\hline & $\mathrm{C2}-2$ & & $4 \Phi 8$ & $5 \Phi 8$ & & $0.48 \%$ \\
\hline & C2-3 & & & $5 \Phi 10$ & & $0.75 \%$ \\
\hline & C2-4 & & & $5 \Phi 12$ & & $1.09 \%$ \\
\hline \multirow{5}{*}{$\begin{array}{l}\text { Gr } \\
\text { ou } \\
\text { D } 3\end{array}$} & C31 & & \multirow{5}{*}{$4 \Phi 10$} & $5 \Phi 6$ & \multirow{5}{*}{$0.50 \%$} & $0.27 \%$ \\
\hline & $\mathrm{C32}$ & & & $5 \Phi 8$ & & $0.48 \%$ \\
\hline & $\mathrm{C33}$ & & & $5 \Phi 10$ & & $0.75 \%$ \\
\hline & $\mathrm{C34}$ & & & $5 \Phi 12$ & & $1.09 \%$ \\
\hline & & & & & & \\
\hline \multirow{4}{*}{$\begin{array}{l}\mathrm{Gr} \\
\text { ou } \\
\mathrm{D} 4\end{array}$} & C41 & & \multirow{4}{*}{$4 \Phi 12$} & $5 \Phi 6$ & \multirow{4}{*}{$0.73 \%$} & $0.27 \%$ \\
\hline & C52 & & & $5 \Phi 8$ & & $0.48 \%$ \\
\hline & $\mathrm{C} 43$ & & & $5 \Phi 10$ & & $0.75 \%$ \\
\hline & C44 & & & $5 \Phi 12$ & & 1.09 \\
\hline
\end{tabular}


COMBINED EFFECT OF STIRRUP VOLUMETRIC RATIO WITH VERTICAL STEEL BARS ON THE BEHAVIOR OF R.C.

COLUMNS UNDER AXIAL LOADS

Table 2. Concrete mix design

\begin{tabular}{|c|c|}
\hline Constituents & Mix proportions by weight for m3 \\
\hline Crushed stone & $1108 \mathrm{~kg}$ \\
\hline Gradate sand & $640 \mathrm{~kg}$ \\
\hline Water & $135 \mathrm{lit}$ \\
\hline Cement & $270 \mathrm{~kg}$ \\
\hline Water/cement ratio (w/c) & $50 \%$ \\
\hline
\end{tabular}
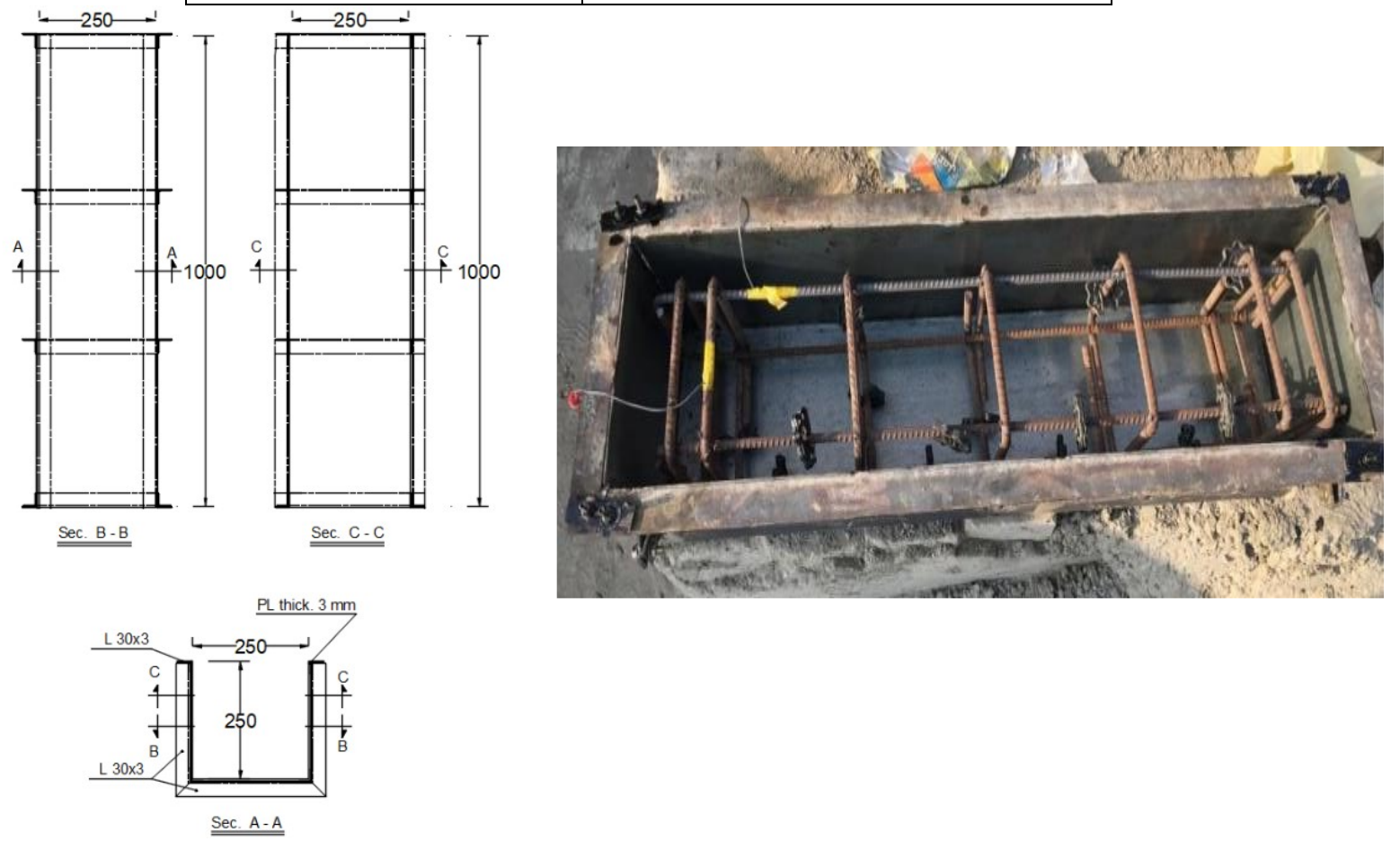

Fig. 2 Steel forms for casting

\subsubsection{Strain gauges locations}

- Tow strain gauges have been mounted on the vertical reinforcement bars and steel stirrups. One was mounted on the corner vertical steel bars, while another one was mounted on the steel stirrups as shown in Fig. [3]. The strain gauges used were manufactured by TOKYO SOKKI KENKYUJO CO. LYD. The strain gauges type used was PFL-30-11-3L, having resistance of $120.4 \pm 0.5 \mathrm{nd} \% \mathrm{Ohms}$ at $11^{\circ} \mathrm{C}$. The gauge factor ranges $2.13 \pm 1.0 \%$.

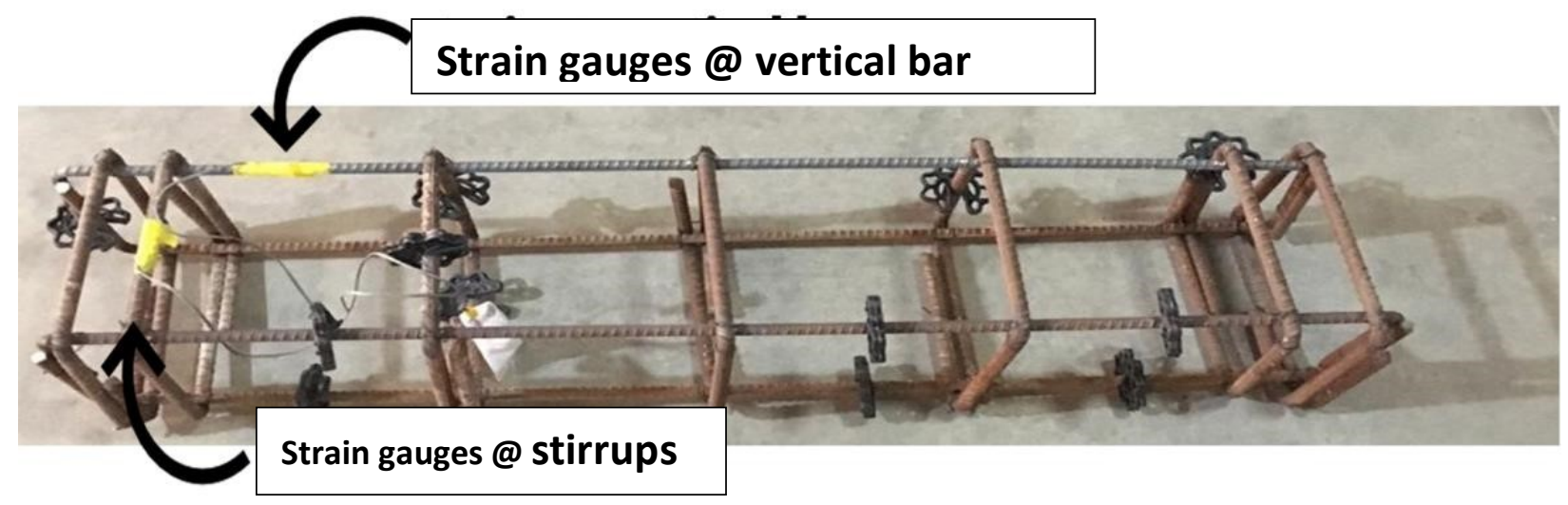

Fig. 3 Location of strain gauges 


\subsubsection{Casting of columns}

- All specimens were casted in steel forms as shown in Fig. [ $\{$ ] and a mechanical vibrator was used. Columns forms were removed and columns specimens were cured.

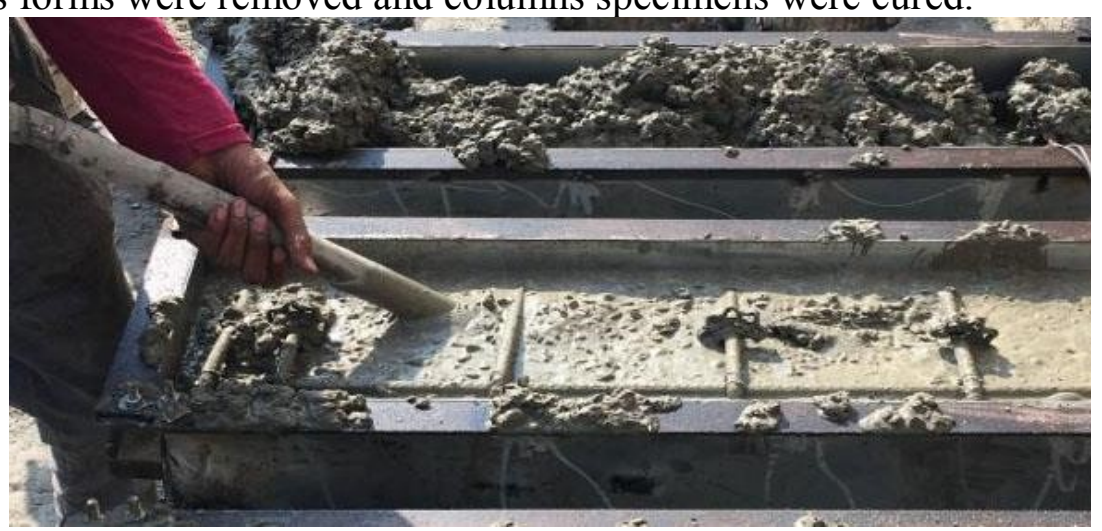

Fig.4 Casting of columns

\subsection{Testing setup and procedure}

All specimens were tested under static loads using the testing machine mounted on the material laboratory of Al-azhar University which has an ultimate compressive load capacity of $2000 \mathrm{kN}$ as shown in Fig. [०]. the readings of loads and strains in reinforcement were recorded through the data acquisition system. The data acquisition system consisted of a laptop computer, a Keithley-500a data acquisition system and the lab tech notebook software package.

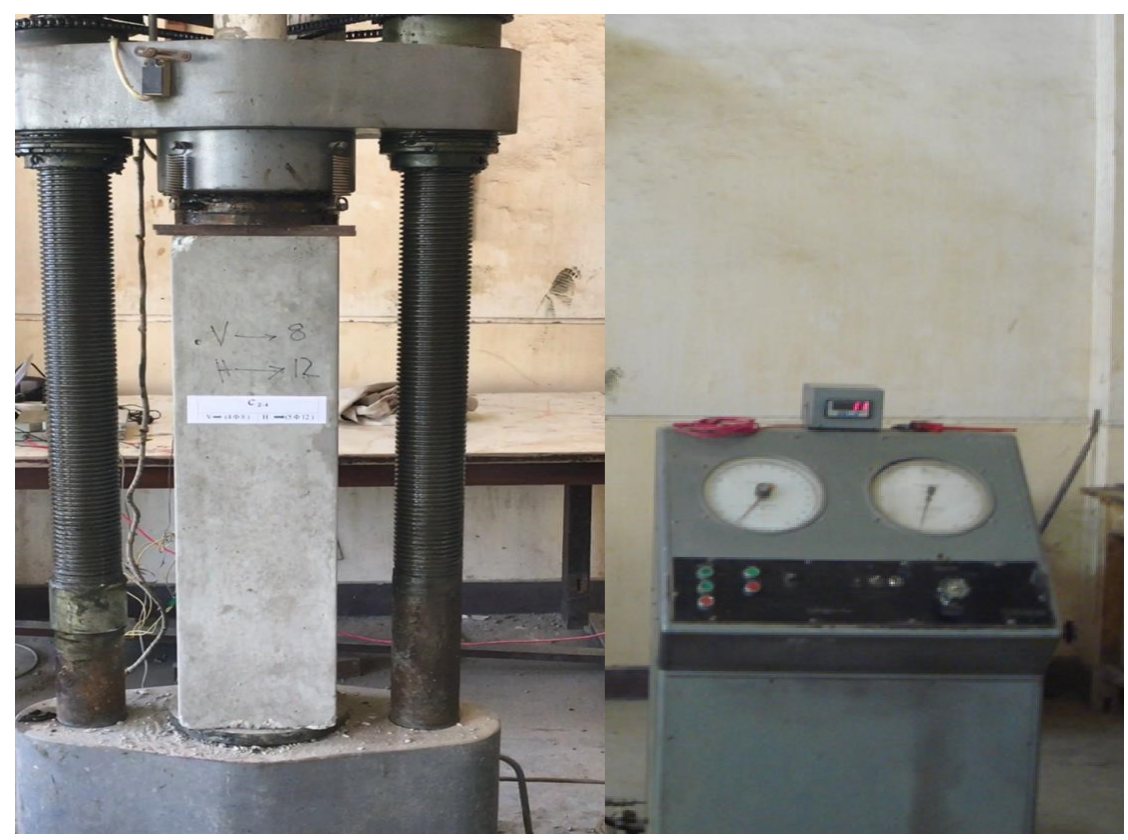

\subsection{Experimental test results}

Fig. 5Test set up

A comparison between the results has been done to investigate the effect of using different vertical steel bars diameter $6,8,10$ and $12 \mathrm{~mm}$ with different stirrups diameters $6,8,10$ and 12 $\mathrm{mm}$.

\subsubsection{Ultimate columns Capacity}

Table (3) and Figure ( 7 ) show the maximum failure loads and percentage column carrying capacity displayed as follows: 


\section{COMBINED EFFECT OF STIRRUP VOLUMETRIC RATIO WITH VERTICAL STEEL BARS ON THE BEHAVIOR OF R.C. COLUMNS UNDER AXIAL LOADS}

Gruop1: Increase in percentage column carrying capacity $11.8 \%$ from control column with increase in volumetric ratio $(\rho \mathrm{v})$ from $0.27 \%$ up to $1.09 \%$ at vertical reinforcement ration $(\mu)$ $0.18 \%$.

Gruop2: Increase in percentage column carrying capacity from $1.2 \%$ up to $12.5 \%$ with increase in volumetric ratio $(\rho \mathrm{v})$ from $0.27 \%$ up to $1.09 \%$ at vertical reinforcement ration $(\mu)$ $0.32 \%$.

Gruop3: Increase in percentage column carrying capacity from $3.2 \%$ up to $13.4 \%$ with increase in volumetric ratio $(\rho \mathrm{v})$ from $0.27 \%$ up to $1.09 \%$ at vertical reinforcement ration $(\mu)$ $0.50 \%$.

Gruop4: Increase in percentage column carrying capacity from $4.1 \%$ up to $13.3 \%$ with increase in volumetric ratio ( $\rho \mathrm{v}$ ) from $0.27 \%$ up to $1.09 \%$ at vertical reinforcement ration $(\mu)$ $0.73 \%$.

It can be shown from previous experimental results the failure load increase by increasing vertical reinforcement ration $(\mu)$ up to $3.2 \%$, while the failure load increase by increasing stirrups volumetric ratio $(\rho$ v) up to $11.8 \%$.

Table 3. Failure loads and percentage column carrying capacity from Experimental test (EXP)

\begin{tabular}{|c|c|c|c|c|c|c|c|}
\hline & \multirow{2}{*}{$\begin{array}{l}\text { Columns } \\
\text { name }\end{array}$} & \multicolumn{2}{|c|}{$\begin{array}{l}\text { Internal reinforcement } \\
\text { bars diameter }(\mathrm{mm})\end{array}$} & \multirow{2}{*}{$\begin{array}{l}\text { Vertical } \\
\text { steel bar } \\
(\mu) \%\end{array}$} & \multirow{2}{*}{$\begin{array}{l}\text { Stirrup } \\
(\rho \mathrm{v}) \%\end{array}$} & \multirow{2}{*}{$\begin{array}{l}\text { Ultimate } \\
\text { load } \\
\text { (EXP)KN }\end{array}$} & \multirow{2}{*}{$\begin{array}{l}\text { \% Column } \\
\text { carrying } \\
\text { capacity(EXP) }\end{array}$} \\
\hline & & Ver. Bar & stirrups & & & & \\
\hline & C1-1 & \multicolumn{2}{|c|}{ Control Column } & $0.18 \%$ & $0.27 \%$ & 1074.6 & $100.0 \%$ \\
\hline \multirow{4}{*}{$\begin{array}{l}\mathrm{Gr} \\
\text { ou } \\
\text { o } 1\end{array}$} & $\mathrm{C} 1-1$ & \multirow{4}{*}{$4 \Phi 6$} & $5 \Phi 6$ & \multirow{4}{*}{$0.18 \%$} & $0.27 \%$ & 1074.6 & $100.0 \%$ \\
\hline & $\mathrm{C} 1-2$ & & $5 \Phi 8$ & & $0.48 \%$ & 1133 & $105.4 \%$ \\
\hline & $\mathrm{C} 1-3$ & & $5 \Phi 10$ & & $0.75 \%$ & 1170 & $108.9 \%$ \\
\hline & $\mathrm{C} 1-4$ & & $5 \Phi 12$ & & $1.09 \%$ & 1201.8 & $111.8 \%$ \\
\hline \multirow{4}{*}{$\begin{array}{l}\mathrm{Gr} \\
\text { ou } \\
\text { o } 2 \\
\end{array}$} & $\mathrm{C} 2-1$ & \multirow{4}{*}{$4 \Phi 8$} & $5 \Phi 6$ & \multirow{4}{*}{$0.32 \%$} & $0.27 \%$ & 1088 & $101.2 \%$ \\
\hline & $\mathrm{C} 2-2$ & & $5 \Phi 8$ & & $0.48 \%$ & 1142 & $106.3 \%$ \\
\hline & $\mathrm{C} 2-3$ & & $5 \Phi 10$ & & $0.75 \%$ & 1171.7 & $109.0 \%$ \\
\hline & $\mathrm{C} 2-4$ & & $5 \Phi 12$ & & $1.09 \%$ & 1209 & $112.5 \%$ \\
\hline \multirow{4}{*}{$\begin{array}{l}\text { Gr } \\
\text { ou } \\
\text { p } 3\end{array}$} & $\mathrm{C} 31$ & \multirow{4}{*}{$4 \Phi 10$} & $5 \Phi 6$ & \multirow{4}{*}{$0.50 \%$} & $0.27 \%$ & 1108.7 & $103.2 \%$ \\
\hline & $\mathrm{C} 32$ & & $5 \Phi 8$ & & $0.48 \%$ & 1148 & $106.8 \%$ \\
\hline & $\mathrm{C} 33$ & & $5 \Phi 10$ & & $0.75 \%$ & 1189.3 & $110.7 \%$ \\
\hline & $\mathrm{C} 34$ & & $5 \Phi 12$ & & $1.09 \%$ & 1218.2 & $113.4 \%$ \\
\hline \multirow{4}{*}{$\begin{array}{l}\mathrm{Gr} \\
\text { ou } \\
\text { p } 4\end{array}$} & $\mathrm{C} 41$ & \multirow{4}{*}{$4 \Phi 12$} & $5 \Phi 6$ & \multirow{4}{*}{$0.73 \%$} & $0.27 \%$ & 1118.3 & $104.1 \%$ \\
\hline & $\mathrm{C} 52$ & & $5 \Phi 8$ & & $0.48 \%$ & 1150.4 & $107.1 \%$ \\
\hline & $\mathrm{C} 43$ & & $5 \Phi 10$ & & $0.75 \%$ & 1197.8 & $111.5 \%$ \\
\hline & C44 & & $5 \Phi 12$ & & $1.09 \%$ & 1217 & $113.3 \%$ \\
\hline
\end{tabular}




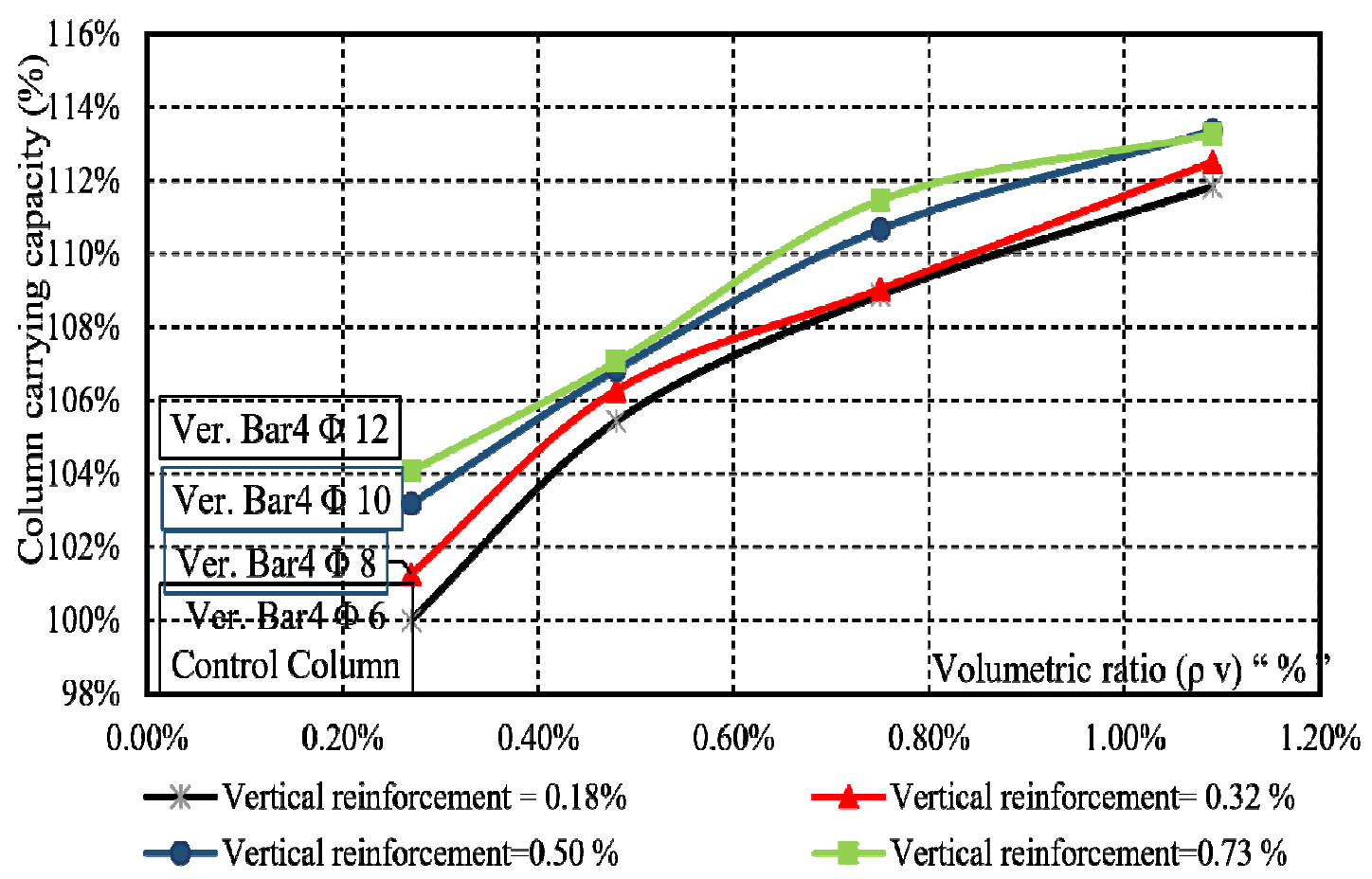

Fig. 6 Relationship between volumetric ratio ( $\rho \mathrm{v})$ and percentage of column carrying capacity (EXP) for different vertical reinforcement bars $(\mu)$

\section{4. 2 Effect of the vertical reinforcement bars and stirrups on strain and ultimate capacity of RC columns (EXP)}

Table (4) show the values of stresses and percentage loads in vertical bars and stirrups at ultimate loads by Experimental test. The compression forces in vertical bars were found to be ranging from $(-1.39 \%$ up to $-6.43 \%)$ of the ultimate failure load with increase in the vertical steel bars percentage from $(0.18 \%$ up to $0.73 \%)$. Also, tension force in stirrups were found to be ranging from $(2.27 \%$ up to $9.53 \%)$ with increase the volumetric ratio from $(0.27 \%$ up to 1.09). Figure (7 up to $1 \cdot$ ) show increase in strain percentage with increase in volumetric ratio of stirrups $(\mu)$ more than increase in strain percentage with increase in vertical steel bars percentage $(\rho \mathrm{v})$. Increasing in volumetric ratio of stirrups percentage has more effect on ultimate capacity of RC columns than increasing in the vertical steel bars percentage and ductility enhancement were obtained. 
COMBINED EFFECT OF STIRRUP VOLUMETRIC RATIO WITH VERTICAL STEEL BARS ON THE BEHAVIOR OF R.C. COLUMNS UNDER AXIAL LOADS

Table 4. Vertical bar and stirrups percentage loads from ultimate loads by Experimental test (EXP)

\begin{tabular}{|c|c|c|c|c|c|c|c|c|c|c|}
\hline & & & & & & & \multicolumn{2}{|c|}{ Compression force } & \multicolumn{2}{|c|}{ Tension force } \\
\hline & \multirow[t]{2}{*}{$\begin{array}{c}\text { Columns } \\
\text { name }\end{array}$} & \multicolumn{2}{|c|}{$\begin{array}{c}\text { Internal } \\
\text { reinforcement } \\
\text { bar }\end{array}$} & \multirow[t]{2}{*}{$\begin{array}{l}\text { Ver. bar } \\
\text { ( } \square) \%\end{array}$} & \multirow[t]{2}{*}{$\begin{array}{l}\text { Stirrups } \\
(\rho \text { v) } \%\end{array}$} & \multirow{2}{*}{$\begin{array}{c}\text { Ultimate } \\
\text { load } \\
(\mathrm{EXP}) \\
\mathrm{KN}\end{array}$} & \multirow[t]{2}{*}{$\begin{array}{l}\text { Ver. bar } \\
\text { Stress } \\
\text { N/mm }\end{array}$} & \multirow[t]{2}{*}{$\begin{array}{l}\text { \% ver. bar / } \\
\text { ultimate } \\
\text { loads }\end{array}$} & \multirow[t]{2}{*}{$\begin{array}{l}\text { Stirrup } \\
\text { stress } \\
\mathrm{N} / \mathrm{mm}\end{array}$} & \multirow{2}{*}{$\begin{array}{l}\text { \%Stirrups } \\
\text { load / } \\
\text { ultimate } \\
\text { loads }\end{array}$} \\
\hline & & Ver. & Stirrups & & & & & & & \\
\hline \multirow{4}{*}{ 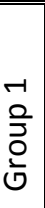 } & $\mathrm{C} 1-1$ & \multirow{4}{*}{$4 \Phi 6$} & $5 \Phi 6$ & \multirow{4}{*}{$0.18 \%$} & $0.27 \%$ & 1074.6 & -629 & $-1.39 \%$ & 822 & $2.27 \%$ \\
\hline & $\mathrm{C} 1-2$ & & $5 \Phi 8$ & & $0.48 \%$ & 1133 & -551 & $-1.16 \%$ & 961 & $4.48 \%$ \\
\hline & $\mathrm{C} 1-3$ & & $5 \Phi 10$ & & $0.75 \%$ & 1170 & -747 & $-1.52 \%$ & 1113 & $7.85 \%$ \\
\hline & $\mathrm{C} 1-4$ & & $5 \Phi 12$ & & $1.09 \%$ & 1201.8 & -770 & $-1.52 \%$ & 753 & $7.44 \%$ \\
\hline \multirow{4}{*}{ 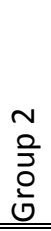 } & $\mathrm{C} 2-1$ & \multirow{4}{*}{$4 \Phi 8$} & $5 \Phi 6$ & \multirow{4}{*}{$0.32 \%$} & $0.27 \%$ & 1088 & -778 & $-3.02 \%$ & 997 & $2.72 \%$ \\
\hline & $\mathrm{C} 2-2$ & & $5 \Phi 8$ & & $0.48 \%$ & 1142 & -804 & $-2.98 \%$ & 1041 & $4.81 \%$ \\
\hline & $\mathrm{C} 2-3$ & & $5 \Phi 10$ & & $0.75 \%$ & 1171.7 & -802 & $-2.89 \%$ & 601 & $4.23 \%$ \\
\hline & $\mathrm{C} 2-4$ & & $5 \Phi 12$ & & $1.09 \%$ & 1209 & -726 & $-2.54 \%$ & 505 & $4.96 \%$ \\
\hline \multirow{4}{*}{ 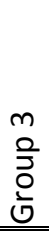 } & $\mathrm{C} 31$ & \multirow{4}{*}{$4 \Phi 10$} & $5 \Phi 6$ & \multirow{4}{*}{$0.50 \%$} & $0.27 \%$ & 1108.7 & -750 & $-4.46 \%$ & 620 & $1.66 \%$ \\
\hline & $\mathrm{C} 32$ & & $5 \Phi 8$ & & $0.48 \%$ & 1148 & -768 & $-4.42 \%$ & 963 & $4.43 \%$ \\
\hline & $\mathrm{C} 33$ & & $5 \Phi 10$ & & $0.75 \%$ & 1189.3 & -875 & $-4.87 \%$ & 640 & $4.45 \%$ \\
\hline & C34 & & $5 \Phi 12$ & & $1.09 \%$ & 1218.2 & -635 & $-3.44 \%$ & 600 & $5.85 \%$ \\
\hline \multirow{4}{*}{ 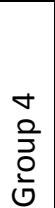 } & $\mathrm{C} 41$ & \multirow{4}{*}{$4 \Phi 12$} & $5 \Phi 6$ & \multirow{4}{*}{$0.73 \%$} & $0.27 \%$ & 1118.3 & -520 & $-4.42 \%$ & 749 & $1.99 \%$ \\
\hline & $\mathrm{C} 42$ & & $5 \Phi 8$ & & $0.48 \%$ & 1150.4 & -748 & $-6.18 \%$ & 889 & $4.08 \%$ \\
\hline & $\mathrm{C} 43$ & & $5 \Phi 10$ & & $0.75 \%$ & 1197.8 & -811 & $-6.43 \%$ & 876 & $6.03 \%$ \\
\hline & $\mathrm{C} 44$ & & $5 \Phi 12$ & & $1.09 \%$ & 1217 & -684 & $-5.34 \%$ & 977 & $9.53 \%$ \\
\hline
\end{tabular}

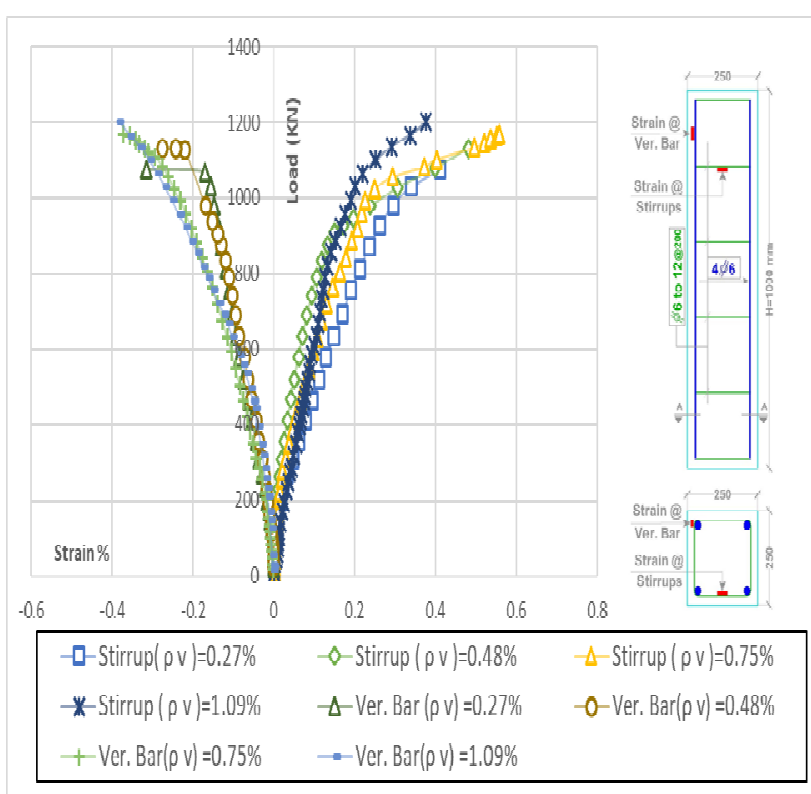

Fig. 7 Comparison between load and strain for group (1) EXP [( $\rho$ v) from $0.27 \%$ up to $1.09 \%$ at vertical reinforcement ration $(\mu) \mathbf{0 . 1 8 \%}$.]

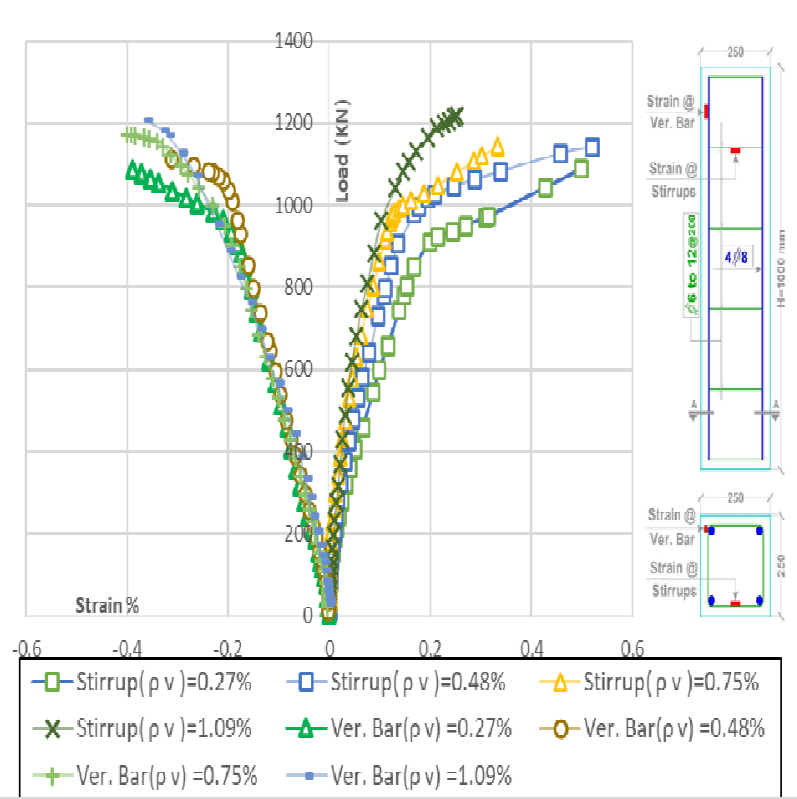

Fig.8 Comparison between load and strain for group (2) $\operatorname{EXP}[$ ( $\rho$ v) from $0.27 \%$ up to $1.09 \%$ at vertical reinforcement ration $(\mu) 0.32 \%$.] 


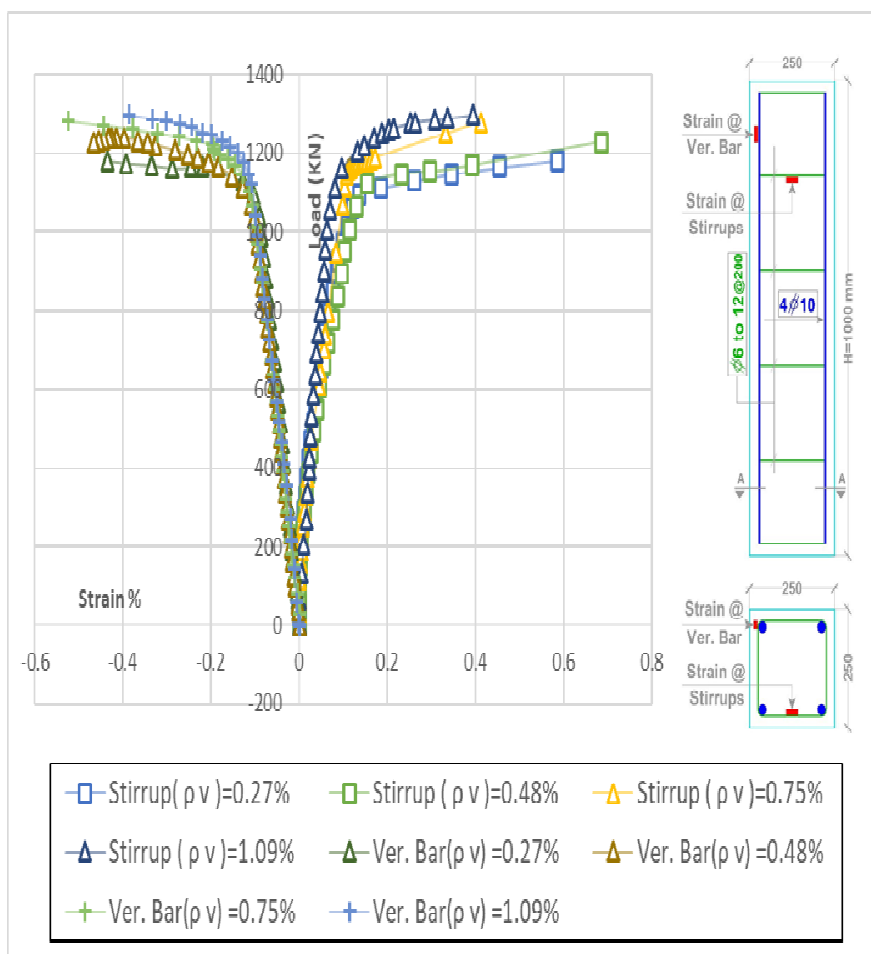

Fig. 9 Comparison between load and strain for group (3) EXP [ ( $\rho$ v) from $0.27 \%$ up to $1.09 \%$ at vertical reinforcement ration $(\mu) \mathbf{0 . 5 0 \%}$.]
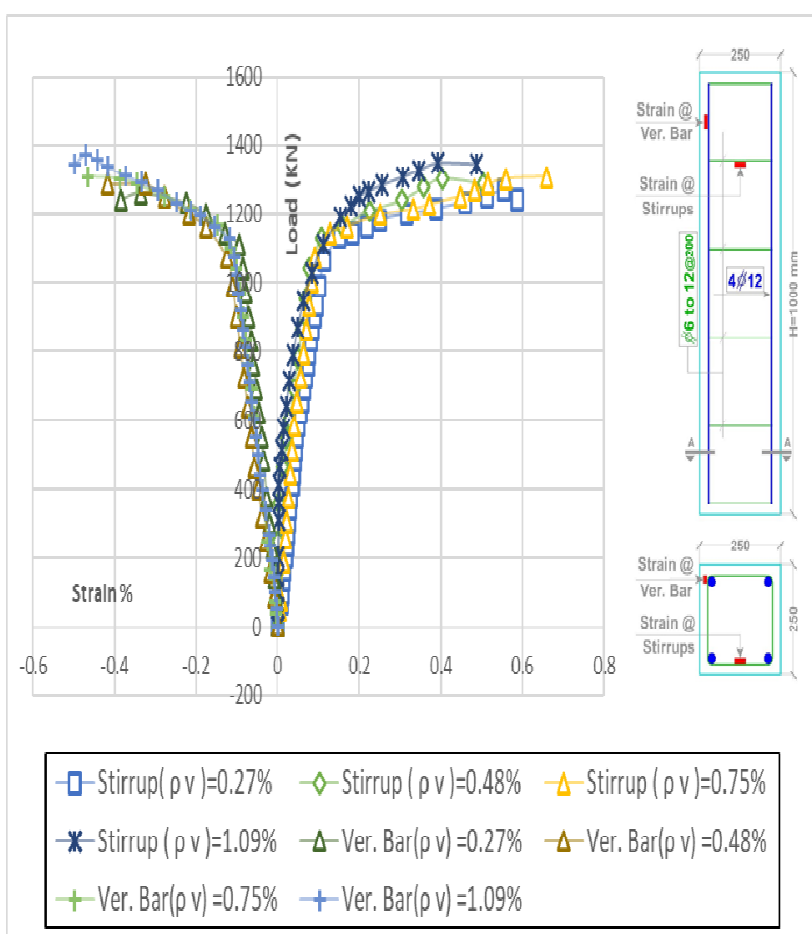

Fig. 10 Comparison between load and strain for group (4) EXP [ ( $\rho$ v) from $0.27 \%$ up to $1.09 \%$ at vertical reinforcement ration $(\mu) 0.73 \%$.]

\section{Finite element analysis}

The finite element package ANSYS 15.0 was used in order to simulate the obtained experimental testing results by introducing a numerical model. The tested columns in the experimental work were modeled to determine the failure loads and strains in each specimen. Comparison of results between experimental and finite element model (FEA) was carried out.

\subsection{Defining element types, real constants, and material properties}

The finite element procedure implemented in this study is developed using the available element types from ANSYS 15.0 element library as illustrated in Table [5]

Table 5. Element types used in ANSYS 15.0 Program

\begin{tabular}{|c|c|c|c|c|c|}
\hline $\begin{array}{c}\text { Material } \\
\text { type }\end{array}$ & $\begin{array}{c}\text { ANSYS } \\
\text { element type }\end{array}$ & Real constant & $\begin{array}{c}\text { Material } \\
\text { numbers }\end{array}$ & Item & Item Constant \\
\hline Concrete & Solid 65 & 1 & 1 & -------- & Solid 65 \\
\hline \multirow{3}{*}{$\begin{array}{c}\text { Vertical bars } \\
\text { \&stirrups }\end{array}$} & \multirow{2}{*}{ Link 180 } & 2 & 2 & Cross-sectional area $(\Phi 6)$ & $28.27\left(\mathrm{~mm}^{2}\right)$ \\
\cline { 3 - 6 } & & 3 & 2 & Cross-sectional area $(\Phi 8)$ & $50.27\left(\mathrm{~mm}^{2}\right)$ \\
\cline { 3 - 6 } & & 5 & 2 & Cross-sectional area $(\Phi ~ 10)$ & $78.5\left(\mathrm{~mm}^{2}\right)$ \\
\hline loading plate & Solid 45 & ------ & 3 & Cross-sectional area $(\Phi ~ 12)$ & $113.1\left(\mathrm{~mm}^{2}\right)$ \\
\hline
\end{tabular}

\subsection{Defining concrete Properties}

The Young's modulus for concrete was taken 19677 (N/mm2) and Poisson's ratio was assumed to be (0.2). Additional concrete material data needed for (SOLID 65) were the shear transfer coefficients, tensile stresses, and compressive stresses. Typical shear transfer 
coefficients ranges were taken from 0.0 to 1.0 , with 0.0 representing a smooth crack (complete loss of shear transfer) and 1.0 representing a rough crack (no loss of shear transfer). The stress- strain curve was defined for concrete element in "ANSYS" program with $\mathrm{fcu}=20$ $\mathrm{N} / \mathrm{mm} 2$ as shown in Figure [11].

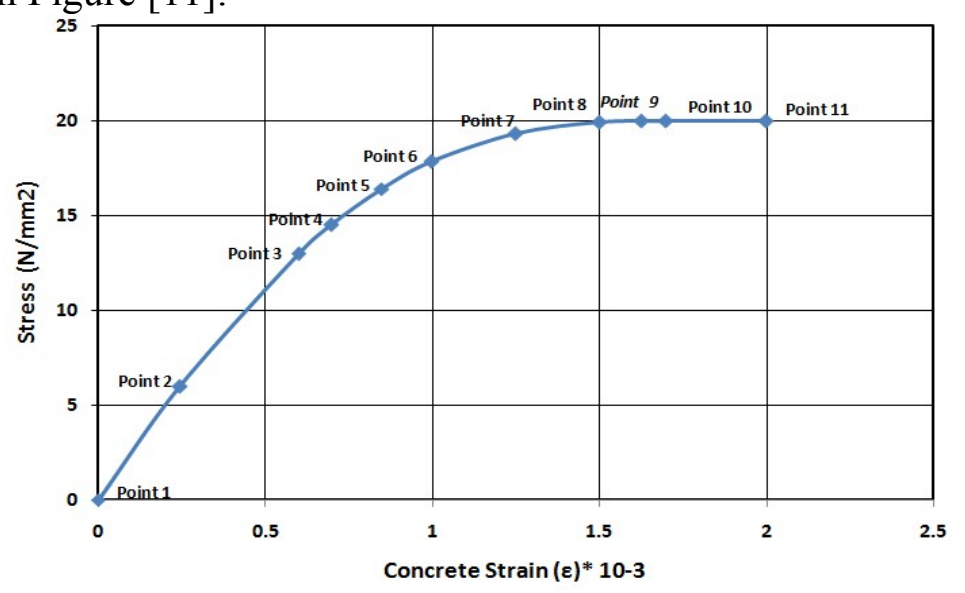

Fig. 11 Concrete stress strain curve (f $\mathrm{cu}=20 \mathrm{~N} / \mathrm{mm} 2)$

\subsection{Defining Of Internal Steel Reinforcement}

The longitudinal and transverse steel is modeled using LINK180 element type. Both yielding and strain hardening failure modes can be accounted. The yield stress, $F y=400(\mathrm{~N} / \mathrm{mm} 2)$. The Young's modulus for reinforcement was taken $2.0 \times 105(\mathrm{~N} / \mathrm{mm} 2)$ and Poisson's ratio was taken to be $(0.3)$. The idealized stress-strain curve for the internal vertical and horizontal reinforcement was used in the finite element model as shown in Figure [12].

\subsection{Defining Of Steel Loading Plate}

The loading plate is modeled using (SOLID45) element type. The Young's modulus for internal steel was taken $2.1 \times 105 \mathrm{~N} / \mathrm{mm} 2$ and Poisson's ratio was assumed to be (0.3).

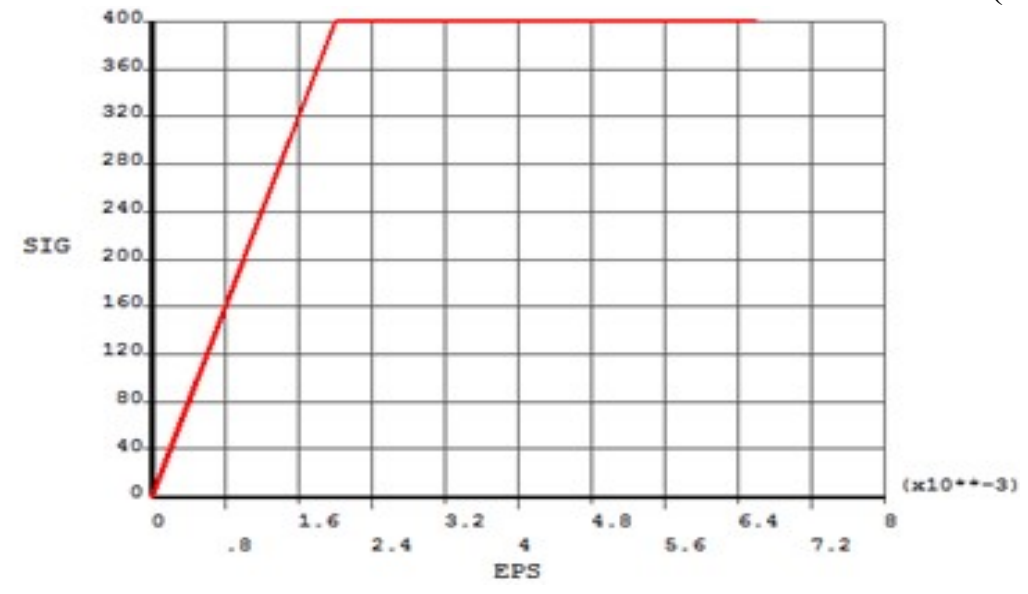

Fig 12. Idealized stress-strain for reinforcing steel bars

\subsection{Boundary Conditions and Loads on Specimen}

Boundary conditions were applied accordingly to simulate the experimental conditions at the base joints of specimen in horizontal translations and rotations in three directions as illustrated in Figure [13]. Axial load is performed on the top of column specimen as axial pressure over the top surface of the column model in (FEA) using "ANSYS15.0" program. A load step option modifier is performed to simulate the axial pressure on the top of column specimen. The incremental of loading is considered with performing the load step option of 
simulating axial load on column specimen. The number of load steps is performed according to the actual load steps applied during the experimental test and depend on the user's definition.

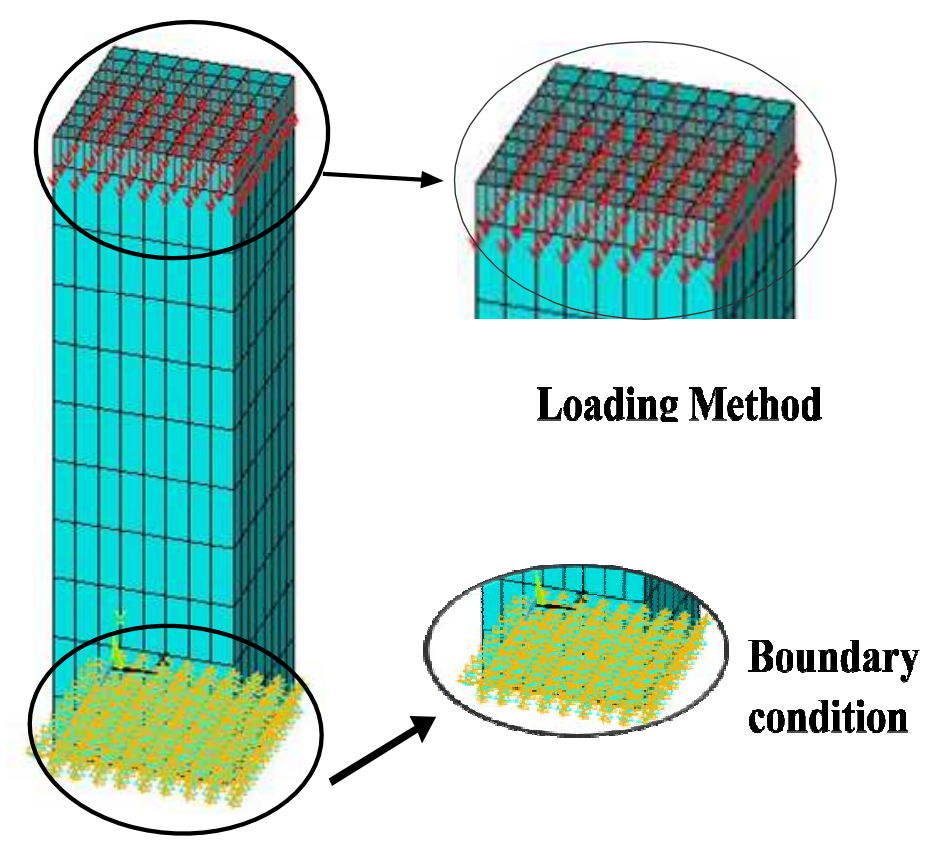

Fig.13 Boundary condition and loading of column specimen

\section{NALYTICAL RESULTS}

\subsection{Ultimate load obtained from FEM analysis}

Table (6) and Figure (14) show the maximum failure loads obtained from FEM analysis and percentage column carrying capacity displayed as follows:

Group 1: Increase in percentage column carrying capacity $12.3 \%$ from control column with increase in volumetric ratio $(\rho \mathrm{v})$ from $0.27 \%$ up to $1.09 \%$ at vertical reinforcement ration $(\mu) 0.18 \%$.

Group 2: Increase in percentage column carrying capacity from $1.0 \%$ up to $13.6 \%$ with increase in volumetric ratio ( $\rho$ v) from $0.27 \%$ up to $1.09 \%$ at vertical reinforcement ration $(\mu) 0.32 \%$.

Group 3: Increase in percentage column carrying capacity from $4.0 \%$ up to $14.3 \%$ with increase in volumetric ratio $(\rho \mathrm{v})$ from $0.27 \%$ up to $1.09 \%$ at vertical reinforcement ration $(\mu) 0.50 \%$.

Group 4: Increase in percentage column carrying capacity from $9.7 \%$ up to $18.6 \%$ with increase in volumetric ratio $(\rho \mathrm{v})$ from $0.27 \%$ up to $1.09 \%$ at vertical reinforcement ration $(\mu) 0.73 \%$.

It can be shown from previous FEM analysis the failure load increase by increasing vertical reinforcement ration $(\mu)$ up to $6.9 \%$, while the failure load increase by increasing stirrups volumetric ratio $(\rho \mathrm{v})$ up to $12.3 \%$. 
COMBINED EFFECT OF STIRRUP VOLUMETRIC RATIO WITH VERTICAL STEEL BARS ON THE BEHAVIOR OF R.C. COLUMNS UNDER AXIAL LOADS

Table 6. Failure loads and percentage column carrying capacity from analytical results (FEM)

\begin{tabular}{|c|c|c|c|c|c|c|c|}
\hline & \multirow{2}{*}{$\begin{array}{l}\text { Columns } \\
\text { name }\end{array}$} & \multicolumn{2}{|c|}{$\begin{array}{c}\text { Internal reinforcement } \\
\text { bars diameter }(\mathrm{mm})\end{array}$} & \multirow{2}{*}{$\begin{array}{c}\text { Vertical } \\
\text { steel bar }(\mu) \quad \%\end{array}$} & \multirow{2}{*}{$\begin{array}{l}\text { Stirrup } \\
(\rho v) \%\end{array}$} & \multirow{2}{*}{$\begin{array}{c}\text { Ultimate } \\
\text { load (EXP) } \\
\text { KN }\end{array}$} & \multirow{2}{*}{$\begin{array}{c}\% \text { Column } \\
\text { carrying capacity } \\
(\mathrm{EXP})\end{array}$} \\
\hline & & Vertical & Stirrups & & & & \\
\hline & C1-1 & \multicolumn{2}{|c|}{ Control Column } & $0.18 \%$ & $0.27 \%$ & 1135 & $100.0 \%$ \\
\hline \multirow{4}{*}{$\begin{array}{l}\text { ㅁ } \\
\text { 을 } \\
\text { 은 }\end{array}$} & C1-1 & \multirow{4}{*}{$4 \Phi 6$} & $5 \Phi 6$ & \multirow{4}{*}{$0.18 \%$} & $0.27 \%$ & 1135 & $100.0 \%$ \\
\hline & C1-2 & & $5 \Phi 8$ & & $0.48 \%$ & 1205 & $106.2 \%$ \\
\hline & $\mathrm{C} 1-3$ & & $5 \Phi 10$ & & $0.75 \%$ & 1236 & $109.0 \%$ \\
\hline & C1-4 & & $5 \Phi 12$ & & $1.09 \%$ & 1254 & $112.3 \%$ \\
\hline \multirow{4}{*}{$\begin{array}{l}\text { N } \\
\frac{2}{7} \\
\text { 인 } \\
\end{array}$} & C2-1 & \multirow{4}{*}{$4 \Phi 8$} & $\overline{5} 5 \Phi 6$ & \multirow{4}{*}{$0.32 \%$} & $0.27 \%$ & 1146 & $101.0 \%$ \\
\hline & $\mathrm{C} 2-2$ & & $5 \Phi 8$ & & $0.48 \%$ & 1184 & $104.3 \%$ \\
\hline & $\mathrm{C} 2-3$ & & $5 \Phi 10$ & & $0.75 \%$ & 1227 & $108.1 \%$ \\
\hline & $\mathrm{C} 2-4$ & & $5 \Phi 12$ & & $1.09 \%$ & 1289 & $113.6 \%$ \\
\hline \multirow{4}{*}{$\begin{array}{l}m \\
\text { 은 } \\
0 \\
\stackrel{0}{0} \\
\end{array}$} & C31 & \multirow{4}{*}{$4 \Phi 10$} & $\overline{5} 5 \Phi 6$ & \multirow{4}{*}{$0.50 \%$} & $0.27 \%$ & 1180 & $104.0 \%$ \\
\hline & C32 & & $5 \Phi 8$ & & $0.48 \%$ & 1229 & $108.3 \%$ \\
\hline & C33 & & $5 \Phi 10$ & & $0.75 \%$ & 1281 & $112.9 \%$ \\
\hline & C34 & & $5 \Phi 12$ & & $1.09 \%$ & 1296 & $114.3 \%$ \\
\hline \multirow{4}{*}{$\begin{array}{l}+ \\
\stackrel{\circ}{7} \\
\text { 인 }\end{array}$} & $\mathrm{C} 41$ & \multirow{4}{*}{$4 \Phi 12$} & $25 \Phi 6$ & \multirow{4}{*}{$0.73 \%$} & $0.27 \%$ & 1244 & $109.7 \%$ \\
\hline & C52 & & $5 \Phi 8$ & & $0.48 \%$ & 1289 & $113.6 \%$ \\
\hline & $\mathrm{C} 43$ & & $5 \Phi 10$ & & $0.75 \%$ & 1309 & $115.4 \%$ \\
\hline & $\mathrm{C} 44$ & & $5 \Phi 12$ & & $1.09 \%$ & 1345 & $118.6 \%$ \\
\hline
\end{tabular}

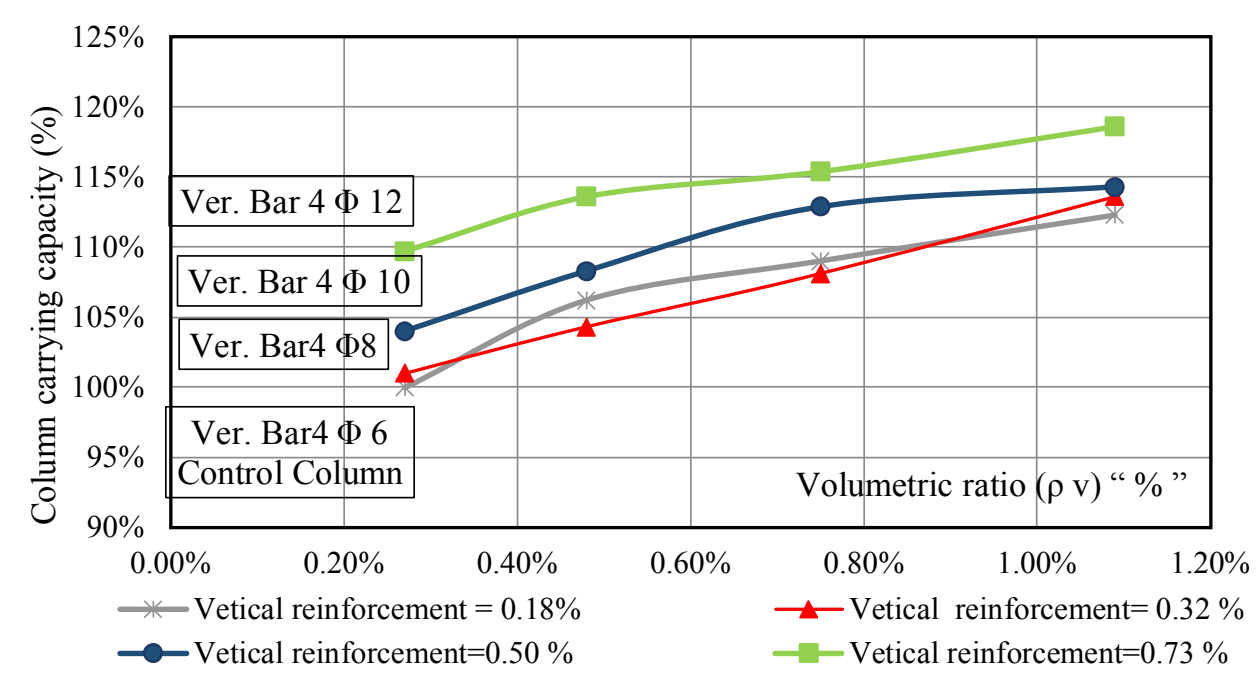

Fig. 14 Relationship between Volumetric ratio and percentage of column carrying capacity (FEM) for different vertical reinforcement bars $(\mu)$ 


\subsection{Effect of the vertical reinforcement bars and stirrups on strain and ultimate capacity of RC columns (FEM)}

Table (7) show the values of stresses and percentage loads in vertical bars and stirrups by analytical results (FEM). The compression forces in vertical bar were found to be ranging from $(-1.44 \%$ up to $-7.03 \%)$ of the ultimate failure with increase the vertical bar percentage from $(0.18 \%$ up to $0.73 \%)$ load. Also, tension force in stirrups were found to be ranging from $(2.36 \%$ up to $8.62 \%)$ with increase in the volumetric ratio from $(0.27 \%$ up to 1.09$)$. Figures (15 up to 18) show increase in strain percentage with increase in volumetric ratio of stirrups ( $\mu$ ) more than increase in strain percentage with increase in vertical steel bars percentage $(\rho$ v). Increasing in volumetric ratio of stirrups percentage has more effect on ultimate capacity of RC columns than increasing in the vertical steel bars percentage and ductility enhancement were obtained.

The results form (FEM) showed the same trend and behavior of the (EXP) results as well fair agreement were obtained.

Table 7. Percentage loads of vertical bar and stirrups from ultimate loads by analytical results (FEM)

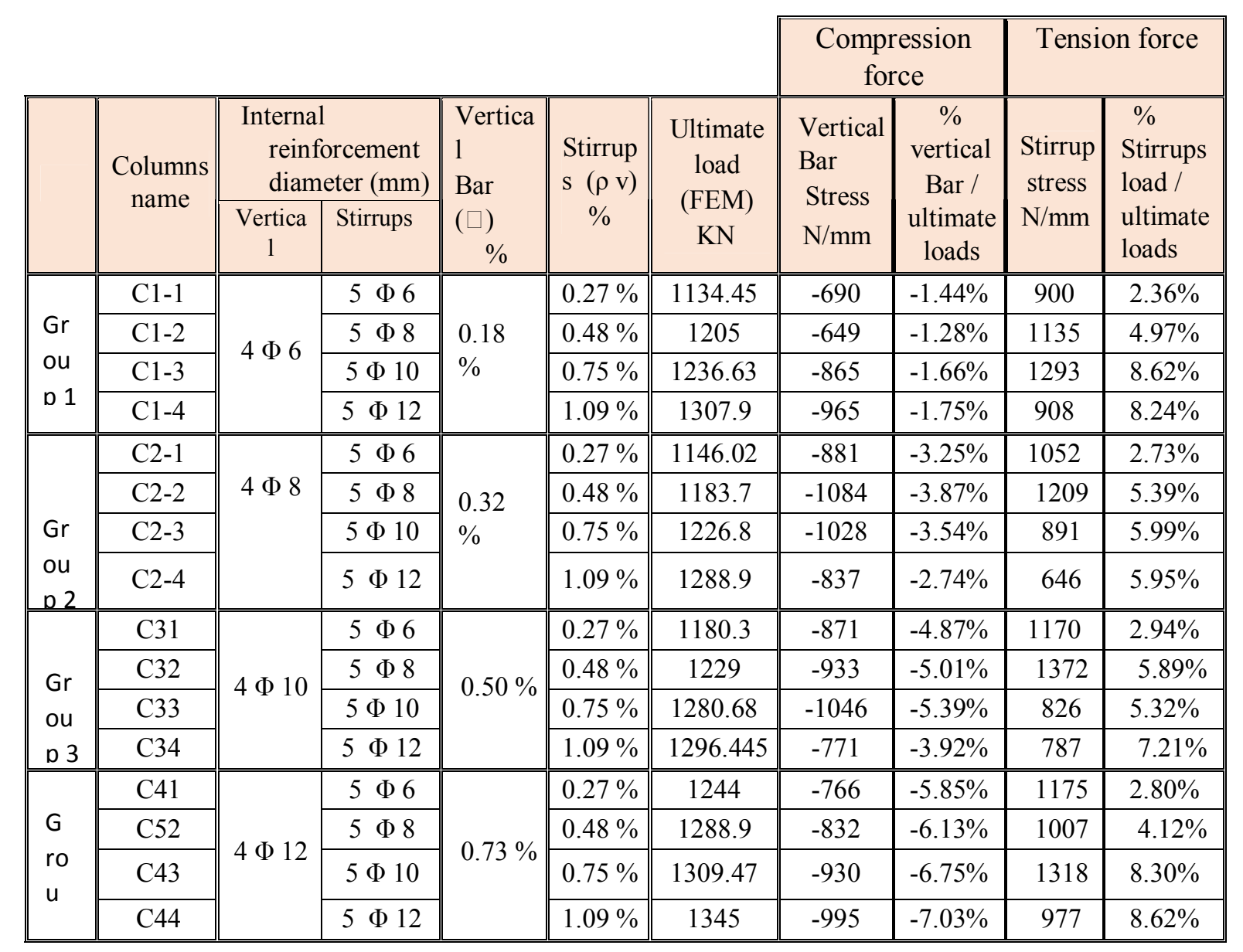




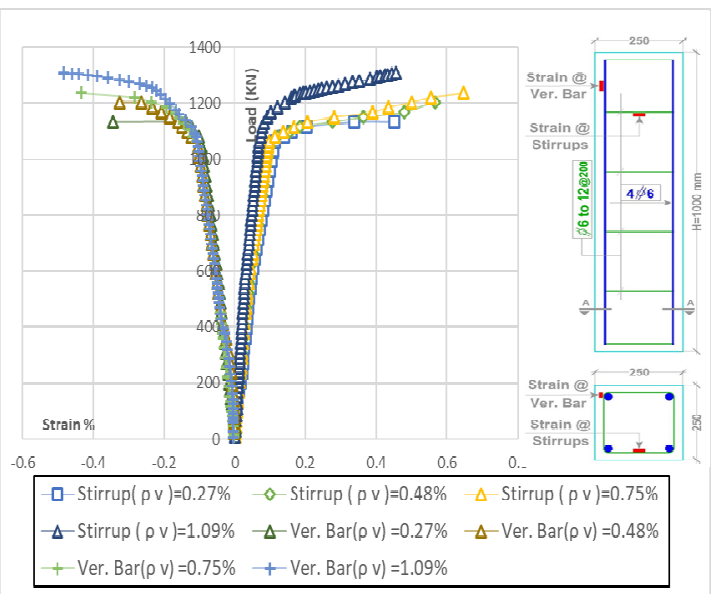

Fig. 15 Comparison between load and strain for group (1)

FEM [ ( $\rho$ v) from $0.27 \%$ up to $1.09 \%$ at vertical reinforcement ration $(\mu) \mathbf{0 . 1 8 \%}$.]

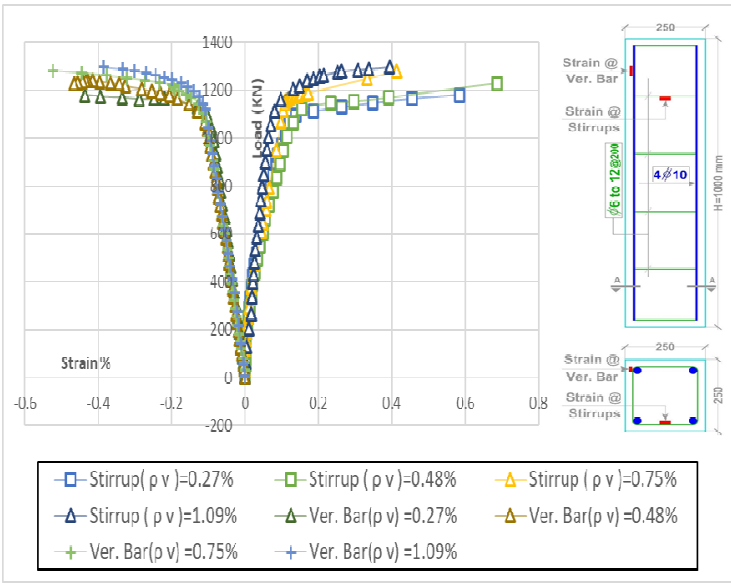

Fig.17 Comparison between load and strain for group (3)

FEM [ $(\rho$ v) from $0.27 \%$ up to $1.09 \%$ at vertical reinforcement ration $(\boldsymbol{\mu})=\mathbf{0 . 5 0 \%}$.]

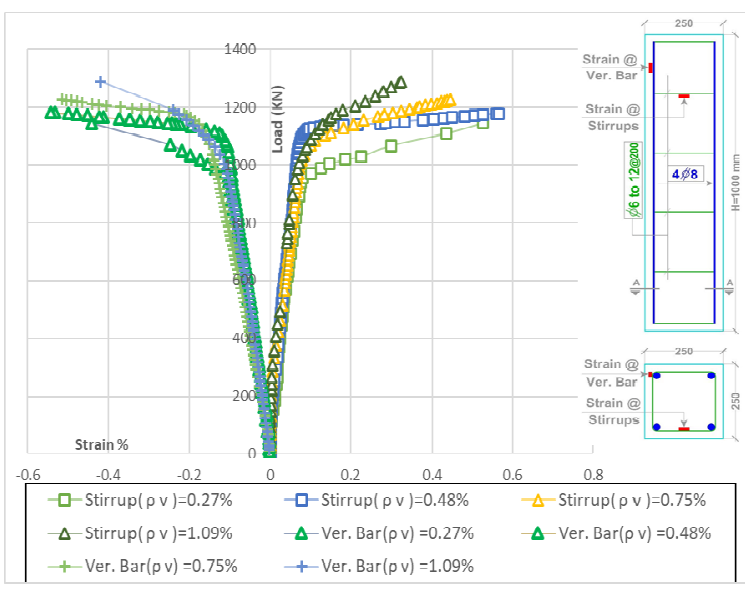

Fig. 16 Comparison between load and strain for group (2) FEM [ $(\rho \mathrm{v})$ from $0.27 \%$ up to $1.09 \%$ at vertical reinforcement ration $(\mu) 0.32 \%$.]

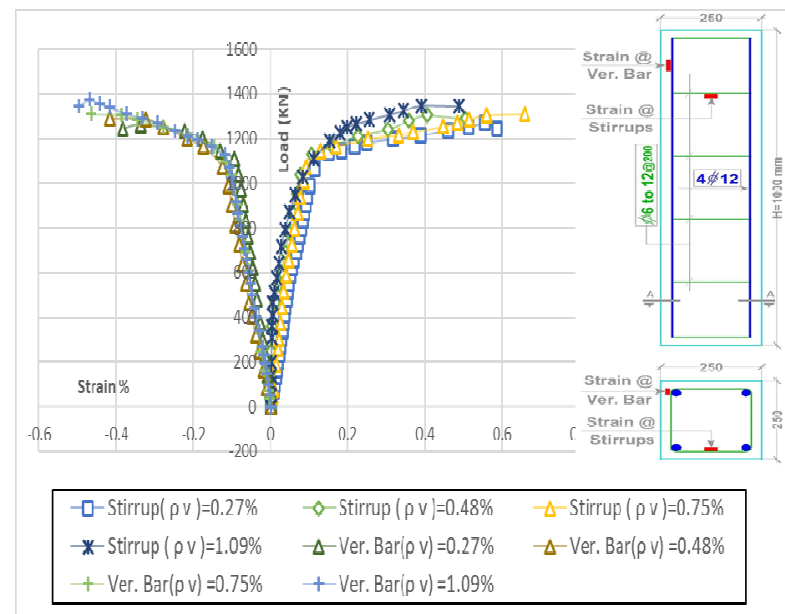

Fig. 18Comparison between load and strain for group

(4) FEM [ $(\rho$ v) from $0.27 \%$ up to $1.09 \%$ at vertical reinforcement ration $(\mu) \mathbf{0 . 7 3 \%}$.]

From the experimental results (EXP) and analytical results (FEM) the percentage of strains in vertical bars and stirrups are explained as follows:

Figures (19 up to 22) show the relationship between loads and percentage of strain from (EXP) and $($ FEM $)$ results for columns at $(\mu)=0.18 \%$ and $(\rho v)=(0.27 \%, 0.48 \%, 0.75 \%$ and $1.09 \%)$

Figures (23 up to 26) show the relationship between loads and percentage of strain from (EXP) and (FEM) results for columns at $(\mu)=0.32 \%$ and $(\rho v)=(0.27 \%, 0.48 \%, 0.75 \%$ and $1.09 \%)$

Figures (27 up to 30) show the relationship between loads and percentage of strain from (EXP) and $(\mathrm{FEM})$ results for columns at $(\mu)=0.50 \%$ and $(\rho \mathrm{v})=(0.27 \%, 0.48 \%, 0.75 \%$ and $1.09 \%)$

Figures (31 up to 34) show the relationship between loads and percentage of strain from (EXP) and (FEM) results for columns at $(\mu)=0.50 \%$ and $(\rho \mathrm{v})=(0.27 \%, 0.48 \%, 0.75 \%$ and $1.09 \%)$

It can be showed from figures (19 to 34) that the obtained percentage of strain from (FEM) has the almost same trend and behavior of (EXP), while the strain values in (FEM) showed an increase in strain values than (EXP). Also it can be shown the strain values increase by increasing vertical reinforcement ratio $(\mu)$ and stirrups volumetric ratio $(\rho$ v). However, fair agreement has been obtained between experimental results and analytical results. 


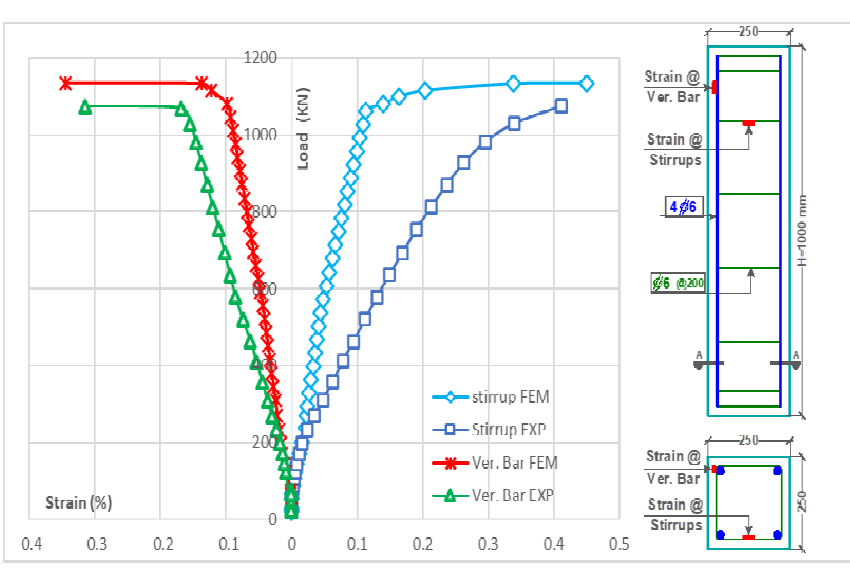

Fig. 19 Relationship between loads and strain from (EXP) and (FEM) results for columns $($ C11) at $(\mu)=$ $0.18 \%$ and

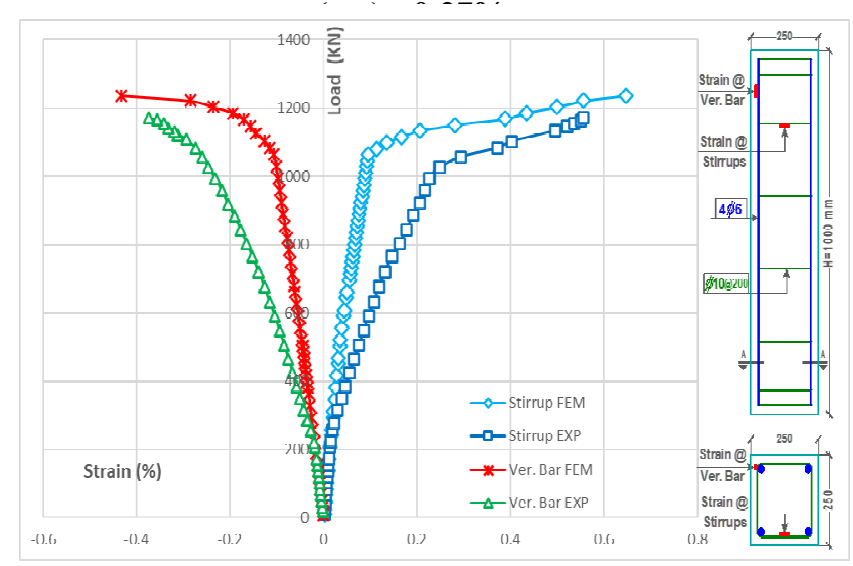

Fig. 21 Relationship between loads and strain from (EXP) and $($ FEM) results for columns $(\mathrm{C13})$ at $(\mu)=0.18 \%$ and $(\rho$ v) $=0 . \vee \diamond \%$

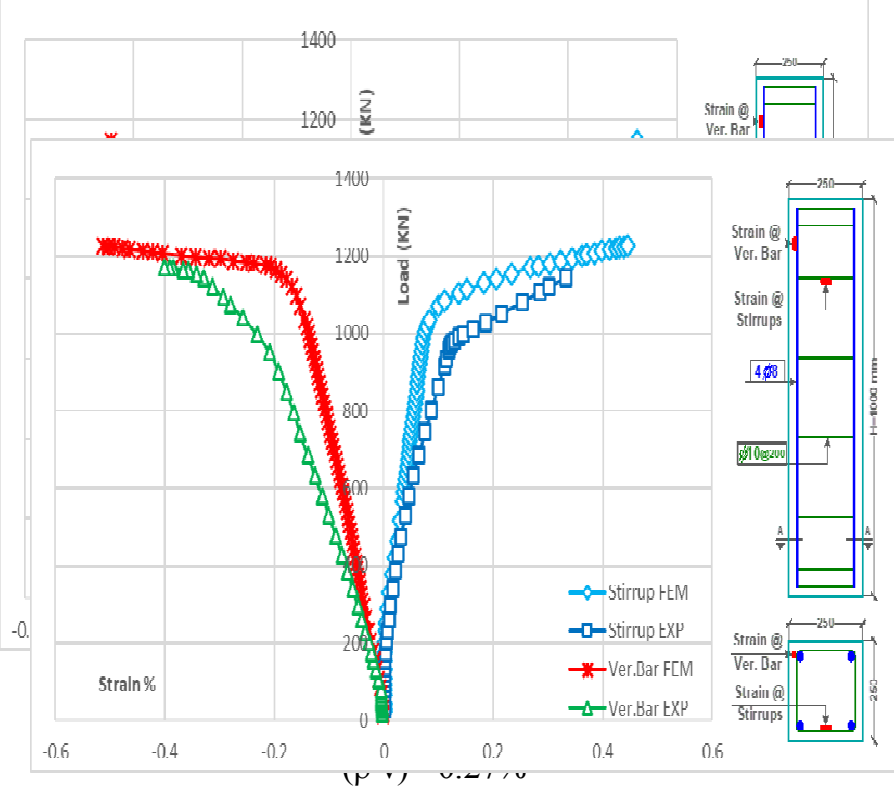

Fig. 25 Relationship between loads and strain from (EXP) and (FEM) results for columns $(\mathrm{C23})$ at $(\mu)=0.32 \%$ and $(\rho \mathrm{v})=\mathbf{0 . 7 5 \%}$

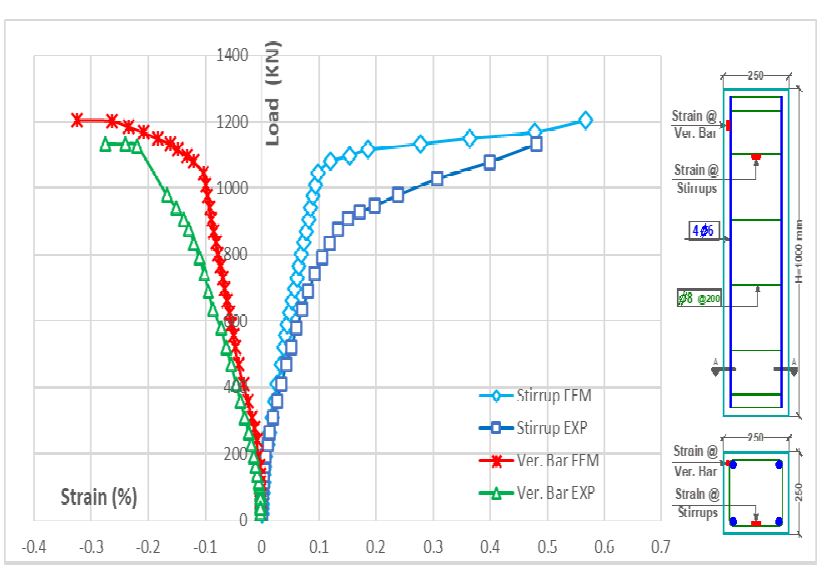

Fig. 20 Relationship between loads and strain from (EXP) and (FEM) results for columns $($ C12) at $(\mu)=$ $0.18 \%$ and (n v) $=\mathbf{0 . 4 8 \%}$

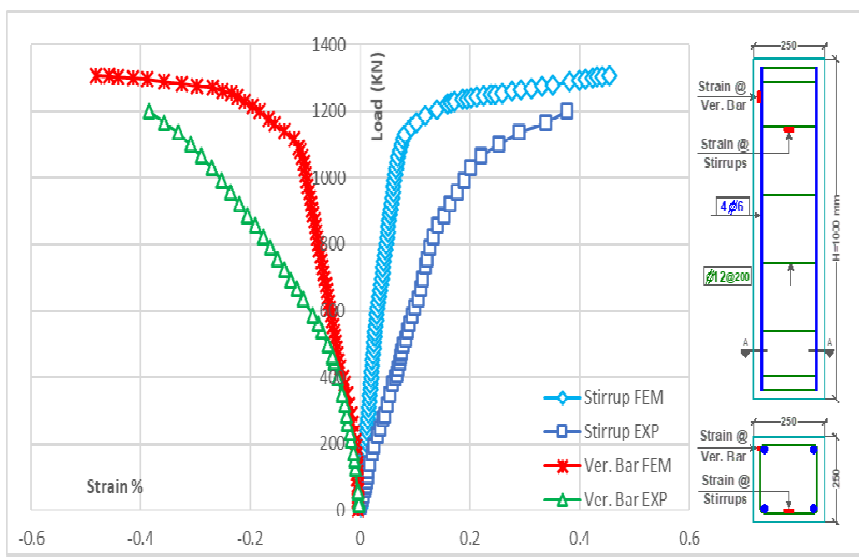

Fig. 22 Relationship between loads and strain from (EXP) and (FEM) results for columns $(\mathrm{C14})$ at $(\mu)=0.18 \%$ and $(\rho \mathrm{v})=1.09 \%$

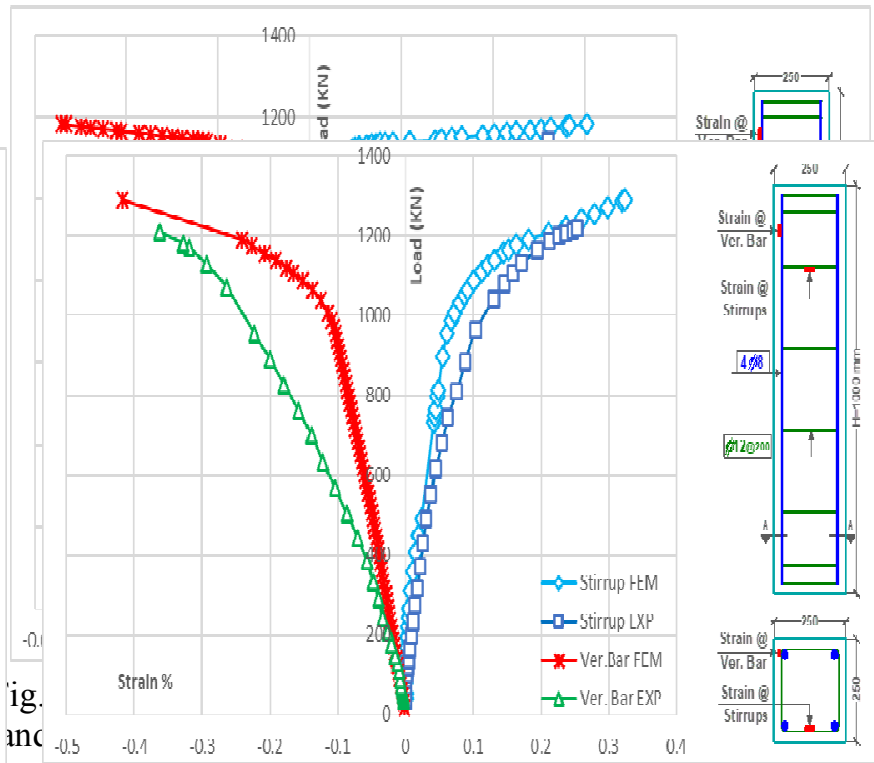

Fig. 26 Relationship between loads and strain from (EXP) and (FEM) results for columns $($ C24) at $(\mu)=0.32 \%$ and $(\rho v)=1.09 \%$ 


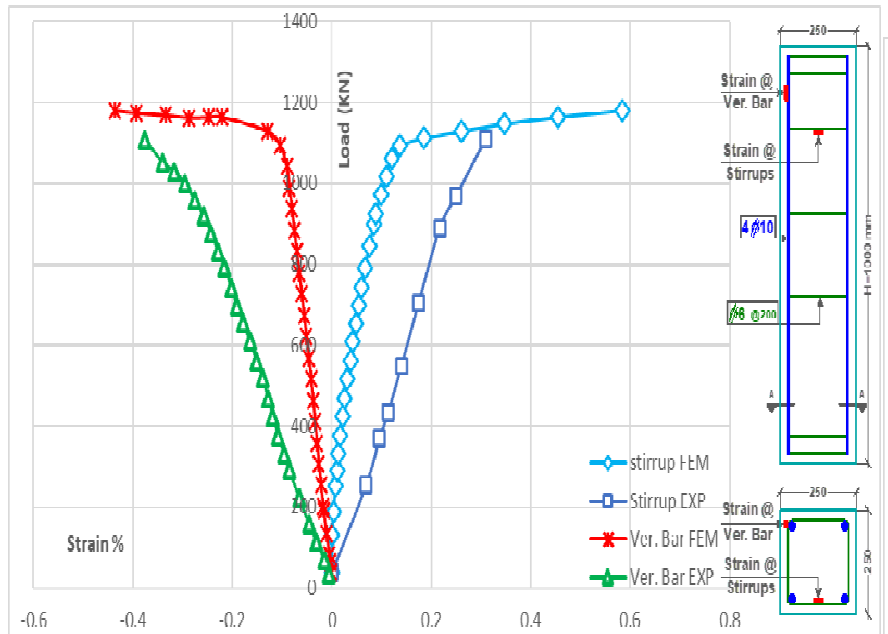

Fig. 27 Relationship between loads and strain from (EXP) and (FEM) results for columns $\left(C^{\top}-\right)$ at $(\mu)=$ $0.50 \%$ and $(\rho$ v $)=0.27 \%$
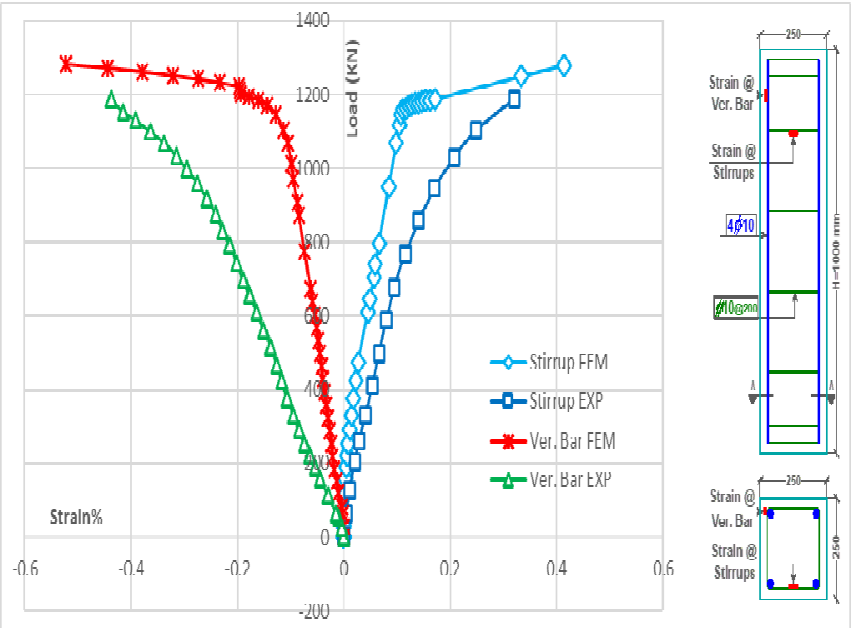

Fig. 29 Relationship between loads and strain from (EXP) and (FEM) results for columns $\left(\mathrm{C}^{\boldsymbol{r} 3}\right)$ at $(\boldsymbol{\mu})=$ $0.50 \%$ and $(\rho \mathrm{v})=\mathbf{0 . 7 5} \%$

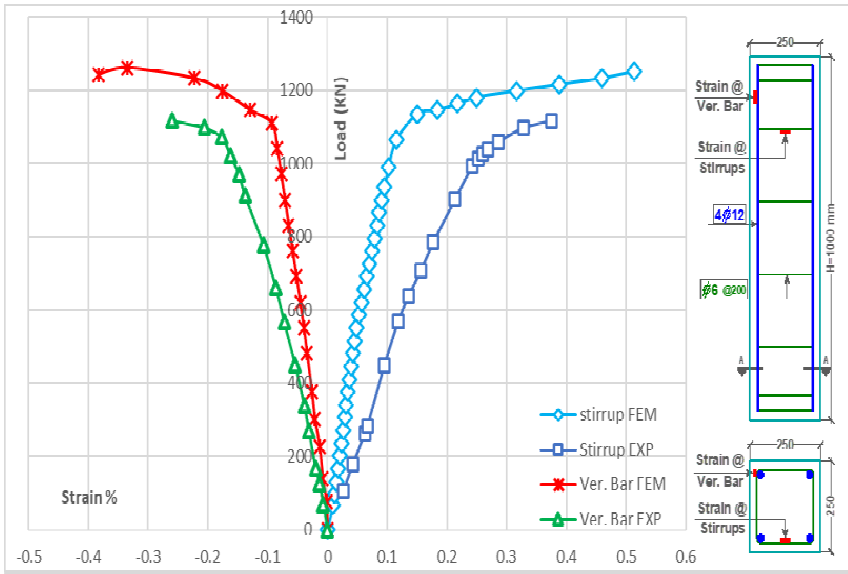

Fig. 31 Relationship between loads and strain from (EXP) and (FEM) results for columns $($ C41) at $(\mu)=$ $0.73 \%$ and $(\rho \mathrm{v})=0.27 \%$

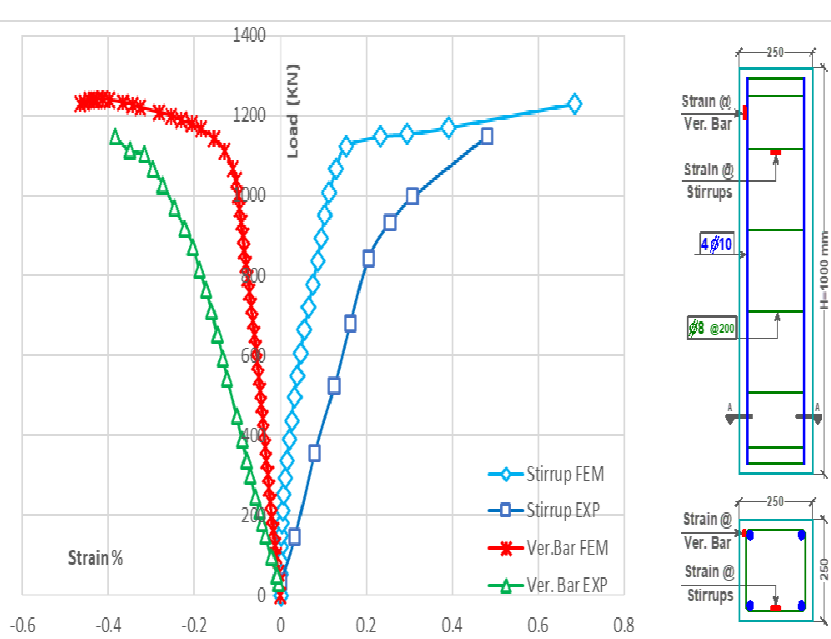

Fig. 28 Relationship between loads and strain from (EXP) and (FEM) results for columns $\left(\mathrm{C}^{\mathrm{r}} 2\right)$ at $(\boldsymbol{\mu})=$ $0.50 \%$ and $(\rho \mathrm{v})=0.48 \%$

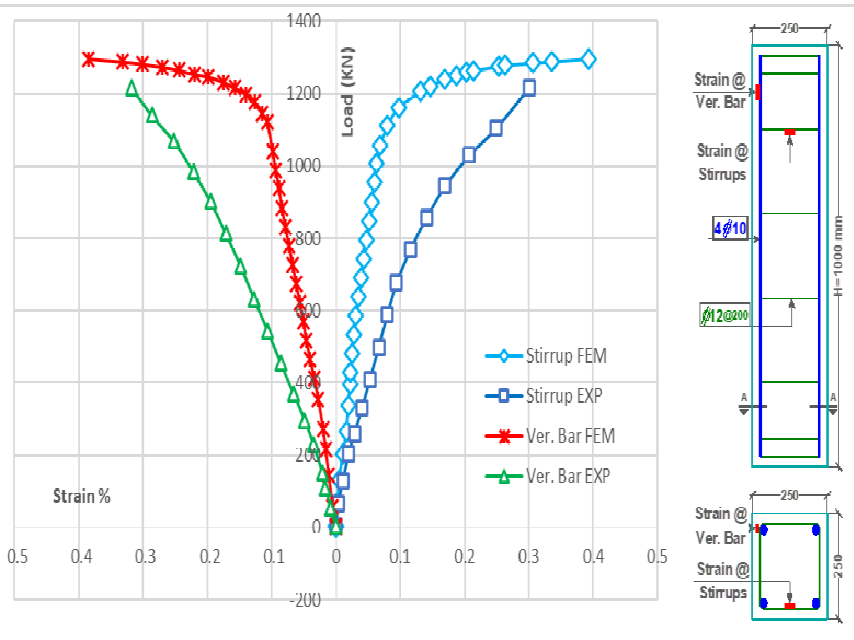

Fig. 30 Relationship between loads and strain from (EXP) and (FEM) results for columns $\left(\mathrm{C}^{\mathrm{r}} 4\right)$ at $(\mu)=$ $0.50 \%$ and $(\rho$ v $)=1.09 \%$

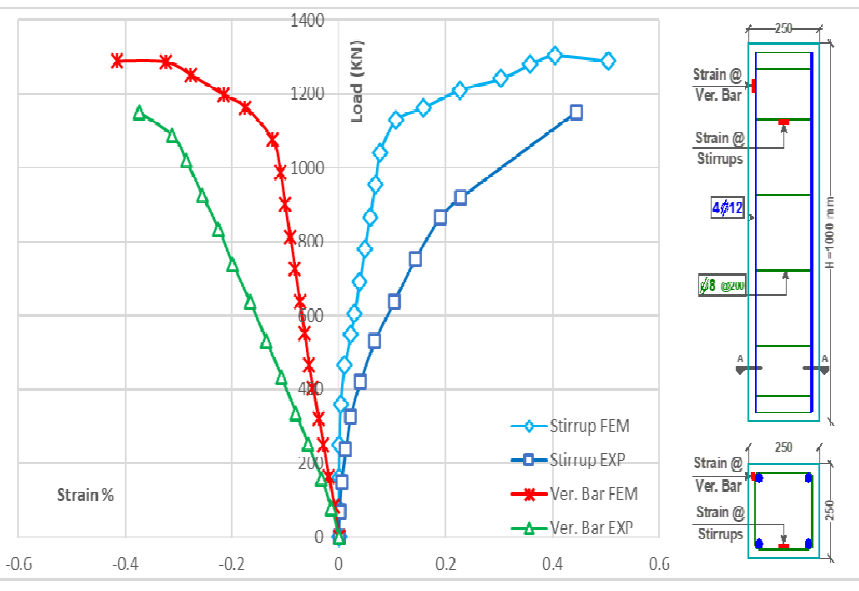

Fig. 32 Relationship between loads and strain from (EXP) and (FEM) results for columns $(\mathrm{C42})$ at $(\mu)=$ $0.73 \%$ and $(\rho \mathrm{v})=0.48 \%$ 


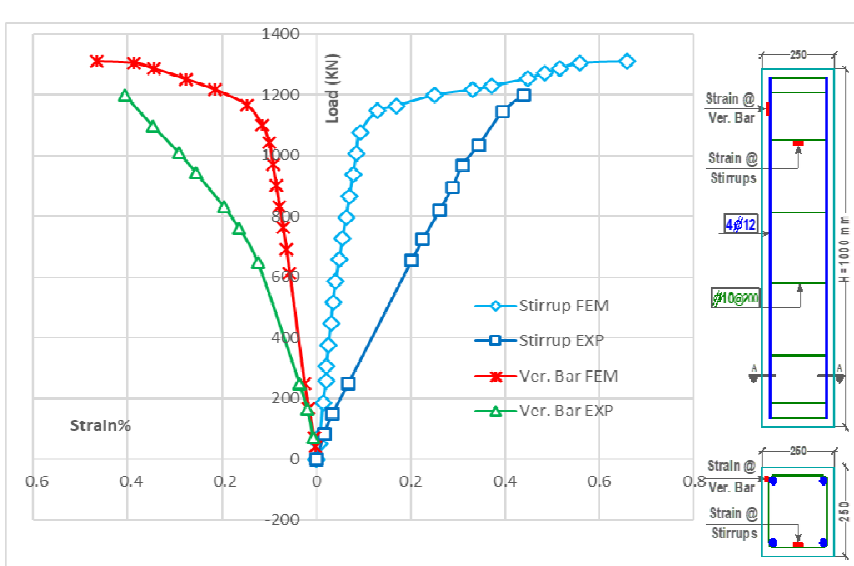

Fig. 33 Relationship between loads and strain from (EXP) and (FEM) results for columns $(\mathrm{C43})$ at $(\mu)=$ $0.73 \%$ and $(\rho \mathrm{v})=0.75 \%$

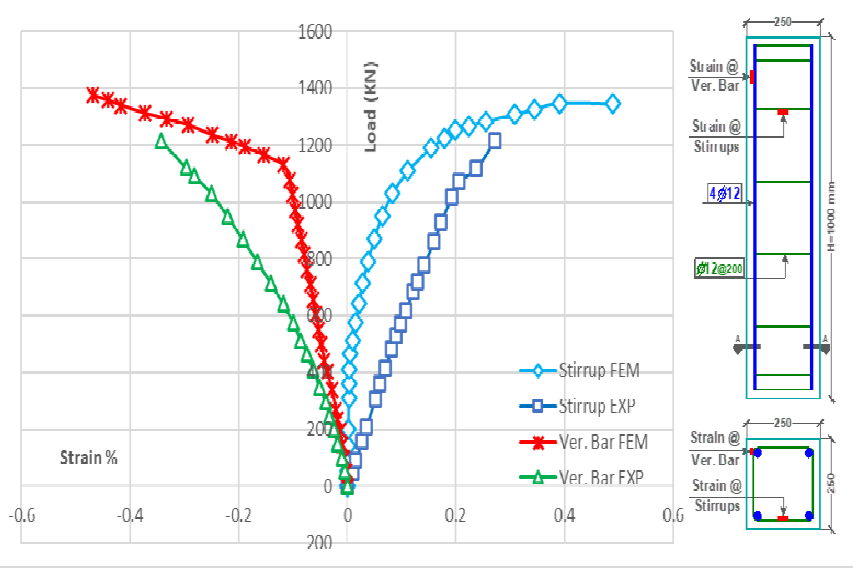

Fig. 34 Relationship between loads and strain from (EXP) and (FEM) results for columns $(\mathrm{C} 44)$ at $(\mu)=$ $0.73 \%$ and $(\rho \mathrm{v})=1.09 \%$

\section{ULTIMATE LOAD OBTAINED FROM FEM ANALYSIS AND EXP RESULTS}

Figures $(35,36$ and 37$)$ show the failure loads from both experimental and analytical results. The obtained comparison showed fair agreement between finite element analysis and experimental results.

\section{FAILURE MODES}

Figure 38 shows the failure modes for RC columns with different vertical steel bars and stirrups volumetric ratio. The failures started by separation of the concrete cover and occur in the upper third of the column height.

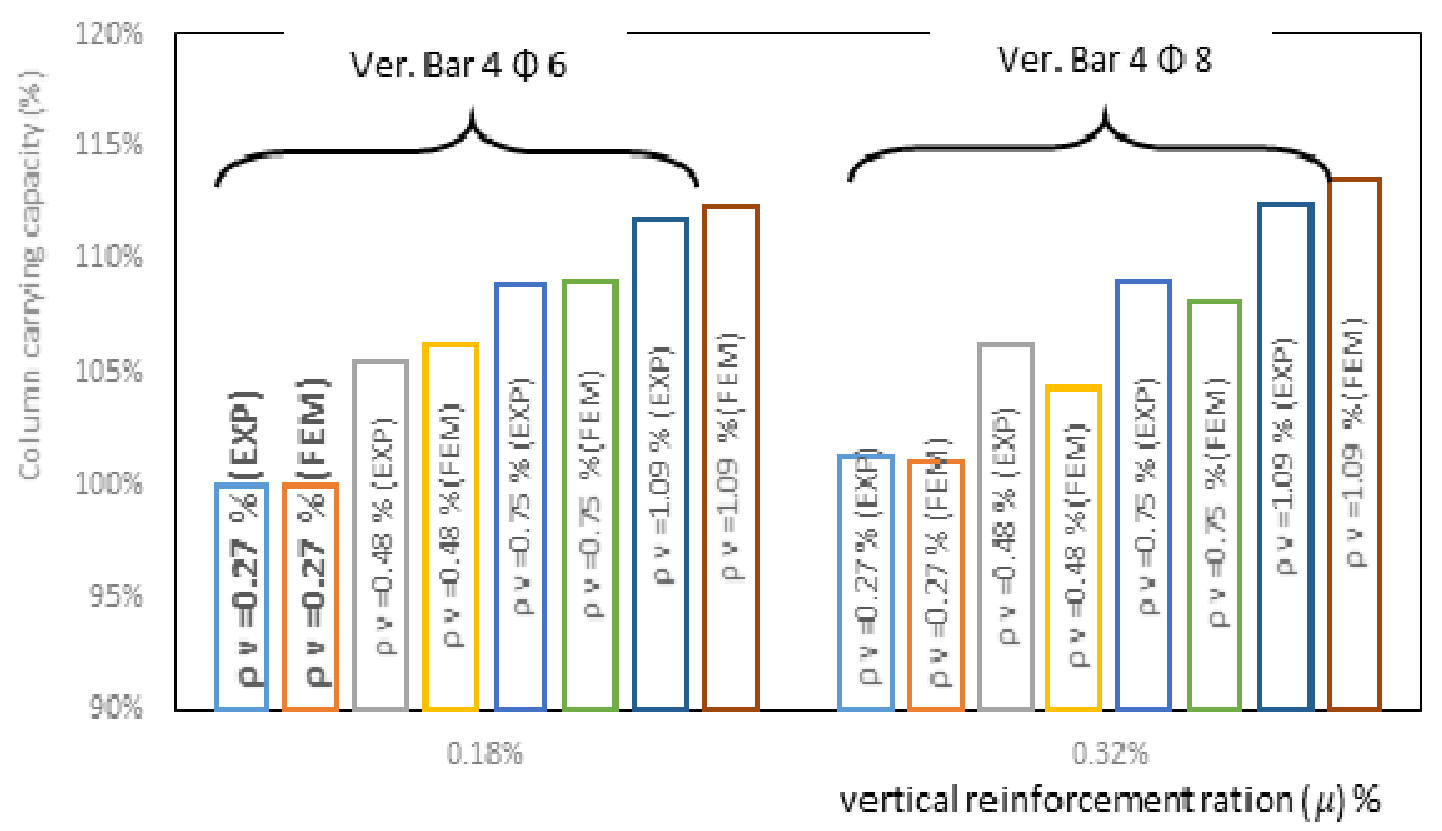

Fig. 35 Relationship between failure load form (EXP) and (FEM) results at stirrups $(\rho \mathrm{v})=0.27 \%$ up to $1.09 \%)$ and vertical reinforcement ration $(\mu)=0.18 \%$ and $0.32 \%$ 
COMBINED EFFECT OF STIRRUP VOLUMETRIC RATIO WITH VERTICAL STEEL BARS ON THE BEHAVIOR OF R.C. COLUMNS UNDER AXIAL LOADS

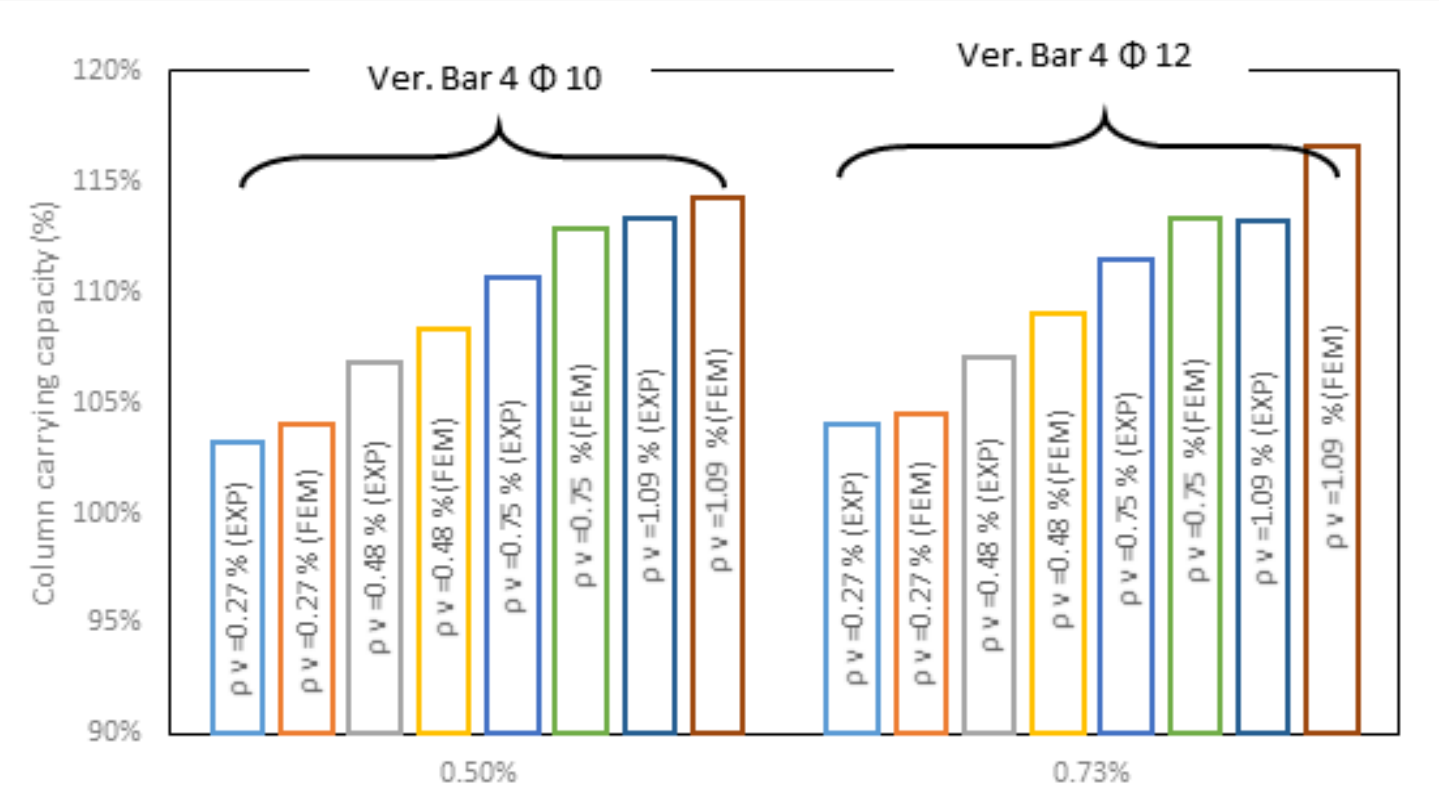

Fig. 36 Relationship between failure load form (EXP) and (FEM) results at stirrups $(\rho$ v) $=0.27 \%$ up to $1.09 \%)$ and vertical reinforcement ration $(\mu)=0.50 \%$ and $0.73 \%$

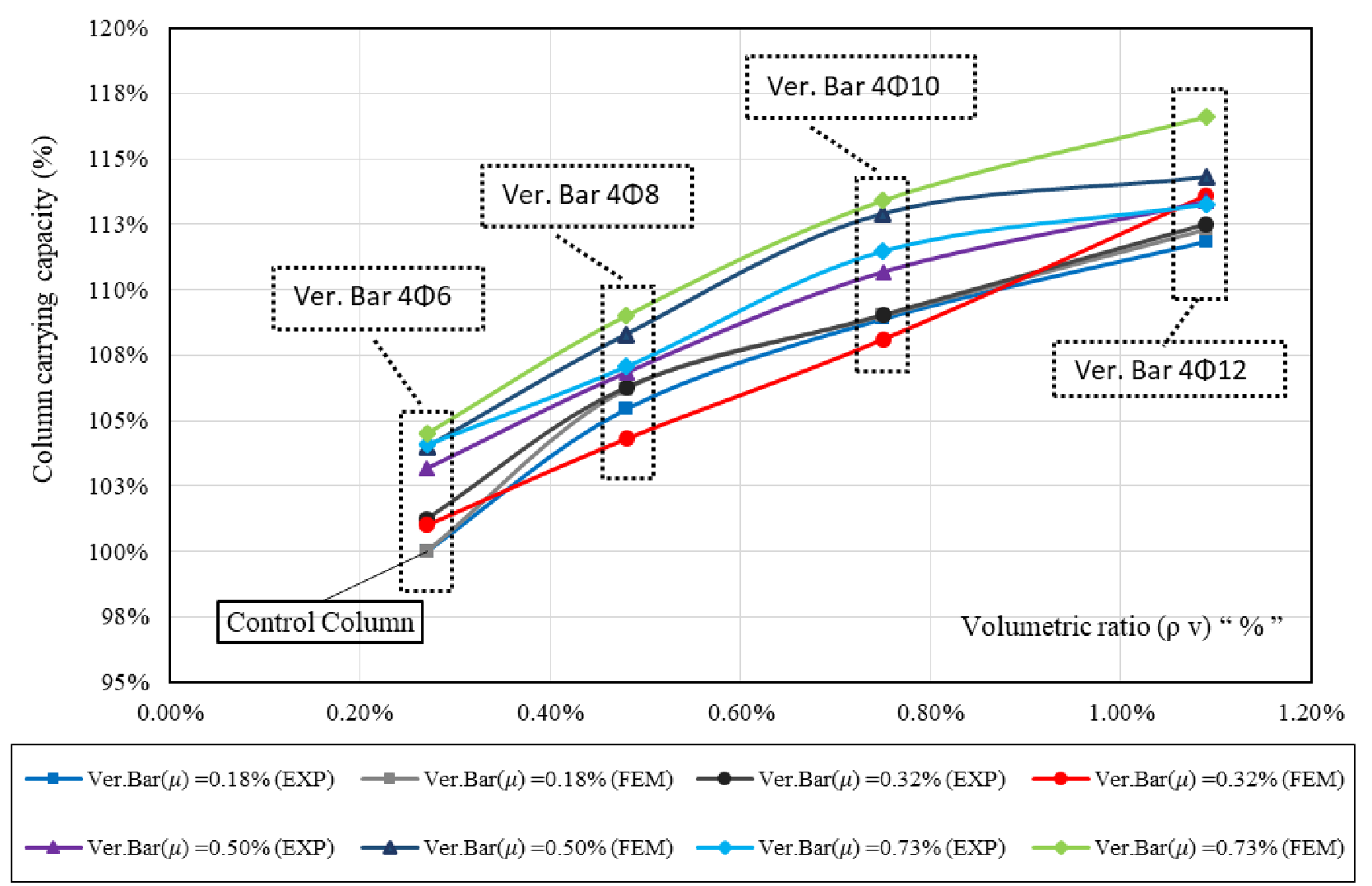

Fig. 37 Relationship between failure load form experimental and analytical finite elements results 


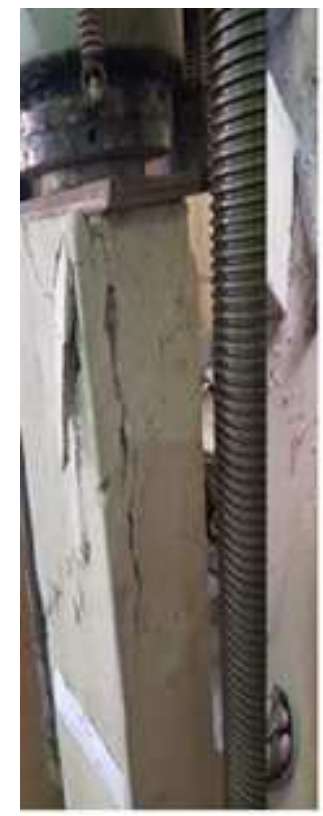

\section{CONCLUSIONS}
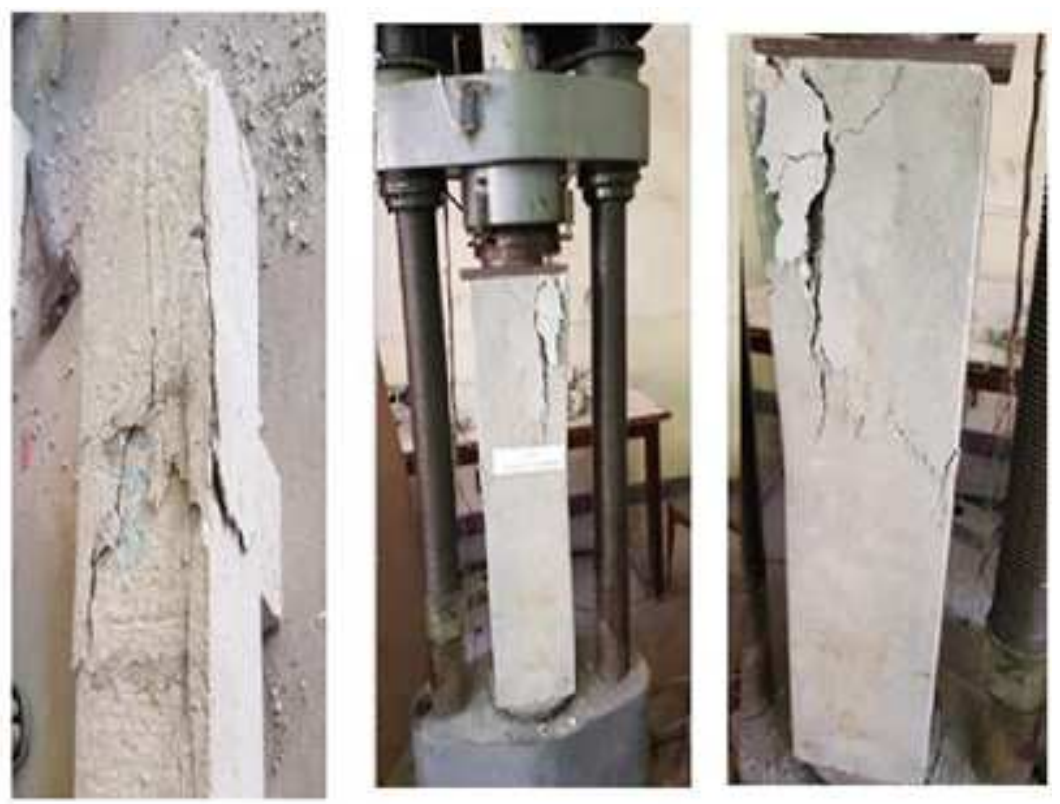

Fig. 38 Failure modes

From the present study, the following conclusions are obtained:

1. From the experimental results the per centage of ultimate failure 16 ad increased by about $4.1 \%$ by increasing percentage of vertical reinforcement from $=(0.18 \%$ up to $0.73 \%$ ) at the same stirrup percentage, while the percentage of the ultimate failure load increased by about $5.7 \%$ from the finite element analysis.

2. From the experimental results the percentage of ultimate failure load increased by $11.8 \%$ with increasing percentage of stirrups from $\rho \mathrm{v}=(0.27 \%$ up to $1.09 \%)$ at the same vertical reinforcement percentage, while the percentage of the ultimate failure load increased by about $12.3 \%$ from the analytical results.

3. Enhancement in ductility is obtained by increasing in stirrups volumetric ratio with less brittle failure of RC columns.

4. The stirrups have a good contribution effect with vertical steel bars on the ultimate load capacity. Thus, it should be taken into consideration the contribution of the concrete, vertical steel bars and stirrups in design of RC columns.

\section{REFERENCES}

1. Cusson, D., \& Paultre, P. (1994), "High-Strength Concrete Columns Confined By Rectangular Ties". Journal of Structural Engineering, 120(3), 783-804.

2. Li, Bing, Park, R. and Tanaka, H. (2001), "Stress-strain behavior of high-strength concrete confined by ultra-high- and normal-strength transverse reinforcements". ACI Structural Journal, V. 98, No. 3, May-June 2001.

3. Yehia A. Daou and Eyad Seifeddin1. (2001), "Minimum lateral reinforcement in reinforced concrete columns". Lebanese Science Journal, Vol. 2, No.2, 2001.

4. J. Němeček, P. Padevět, Z. Bittnar (2004), "Effect of Stirrups on Behavior of Normal and High Strength

5. Hong, K. N., Han, S. H., \& Yi, S. T. (2006), "High-Strength Concrete Columns Confined By LowVolumetric-Ratio Lateral Ties". Engineering Structures, 28(9), 1346-1353.

6. Khaleek, A., Yadav, R. K., \& Rajeev, C. (2012), "Effect Of Lateral Confinement on Strength of Concrete". ISCA Journal of Engineering Sciences, 1(1), 40-44.

7. Wasan Ismail Khalil, Ikbal Naeem Gorgis and Zeinab Raad Mahdi. (2012), "Behavior of high performance fiber reinforced concrete columns" Journal of Engineering and Applied Sciences, Vol. 7, No. 11, November 2012. 
8. Radnic, J., Markic, R., Harapin, A., Matesan, D., \& Baloevic, G. (2013), “ Stirrup Effects On Compressive Strength and Ductility of Confined Concrete Columns" World Journal of Engineering, Vol.10, Issue (6), Pg. 497-506.

9. Xiang Zeng, (2016), "Finite element modelling and analysis of concrete confined by stirrups in square RC columns" The civil engineering journal 3-2016 Article no. 17.

10. Du, M., Jin, L., Du, X., \& Li, D. (2017), "Size Effect Tests of Stocky Reinforced Concrete Columns Confined by Stirrups" .Structural Concrete, Vol.18, Issue (3), pp.454-465.

11. Liu Jin, Zixing Ding, Dong Li, and Xiuli Du, (2018), "Experimental and numerical investigations on the size effect of moderate high-strength reinforced concrete columns under small-eccentric compression" International Journal of Damage Mechanics 2018, Vol. 27(5) 657-685

12. M.K. Abd-Elhamed, M.E. Owida, (2019), "Effect of stirrups densification on ultimate capacity of rectangular reinforced concrete column" Structures Journal 20 (2019) $728-764$ 